\title{
Boundary-Layer Characteristics of Persistent Regional Haze Events and Heavy Haze Days in Eastern China
}

\author{
Peng Huaqing, ${ }^{1}$ Liu Duanyang, ${ }^{2}$ Zhou Bin, ${ }^{1}$ Su Yan, \\ Wu Jiamei, ${ }^{1}$ Shen Hao, ${ }^{1}$ Wei Jiansu, ${ }^{2}$ and Cao $\mathrm{Lu}^{2}$ \\ ${ }^{1}$ Wuxi Meteorological Observatory of Jiangsu Province, Wuxi 214101, China \\ ${ }^{2}$ Observatory of Jiangsu Province, Nanjing 210008, China \\ Correspondence should be addressed to Liu Duanyang; liuduanyang2001@126.com
}

Received 22 March 2015; Revised 1 June 2015; Accepted 4 June 2015

Academic Editor: Xiaofeng Li

Copyright (C) 2016 Peng Huaqing et al. This is an open access article distributed under the Creative Commons Attribution License, which permits unrestricted use, distribution, and reproduction in any medium, provided the original work is properly cited.

\begin{abstract}
This paper analyzed the surface conditions and boundary-layer climate of regional haze events and heavy haze in southern Jiangsu Province in China. There are 5 types with the surface conditions which are equalized pressure (EQP), the advancing edge of a cold front $(\mathrm{ACF})$, the base of high pressure $(\mathrm{BOH})$, the backside of high pressure (BAH), the inverted trough of low pressure (INT), and saddle pressure (SAP) with the haze days. At that time, 4 types are divided with the regional haze events and each of which has a different boundary-layer structure. During heavy haze, the surface mainly experiences EQP, ACF, BOH, BAH, and INT which also have different boundary-layer structures.
\end{abstract}

\section{Introduction}

The people of East China, the Yangtze River Delta, and the Pearl River Delta have been subjected to health risks due to haze since the 21st century, particularly during autumn and winter. The nature of haze is suspended dust particles. In meteorological terms, these particles are called aerosols. Over the last century, many international experts have researched haze [1-15]. In recent years, domestic experts gradually began studying zonal haze and urban haze [16-19].

Regional haze research has also progressed. In China, experts have suggested that the spatial distribution of haze days is expansive in the east and localized in the west; three high-incidence regions in the east were the middle and lower reaches of the Yangtze River, North China, and South China [20-23]. Research has noted distinct transboundary pollution between the cities of the Yangtze River Delta [24]. Experts have analyzed haze and fog in East China and have indicated that haze days gradually increased in most East China cities over the last 30 years $[25,26]$. Song et al. [27] analyzed haze days in Jiangsu Province and indicated that frequent haze days occurred in southern Jiangsu Province due to economic development and urbanization. By analyzing the haze of the
Pearl River Delta, experts found that the regional haze process was related to the airflow stagnation zone, where pollutants accumulate in the boundary layer; thus, pollution becomes highly concentrated near the surface to form haze [28-30]. Regional haze in Beijing, Tianjin, and Hebei has also been researched by Zhao et al. [31] and Wang et al. [32] .

Because haze is greatly influenced by weather, many experts have analyzed the synoptic backgrounds in these cases. Ren et al. [33] suggested that the high-pressure equalizing zone has a cumulative effect on pollutants when researching weather systems and atmospheric pollution of the boundary layer. Rao et al. [34] indicate that forward trough structure and inversion layer provide favorable conditions for regional haze in East-Central China. Zhang et al. [35] noted that the background climate plays an active role in haze formation in autumn, when the haze significantly increases. Many experts have discussed the main causes of haze in terms of low visibility and weather/climate [36-43].

As research on haze has progressed in recent years, the meteorological features of the boundary layer during haze have gradually become an important research direction. $\mathrm{Xu}$ et al. [44] suggested that a thick temperature inversion layer occurred near the surface on haze days. Within this layer, 


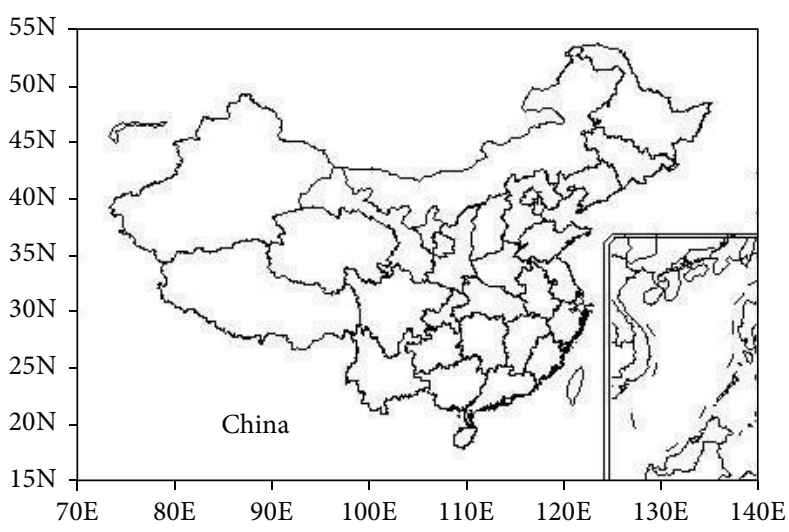

(a)

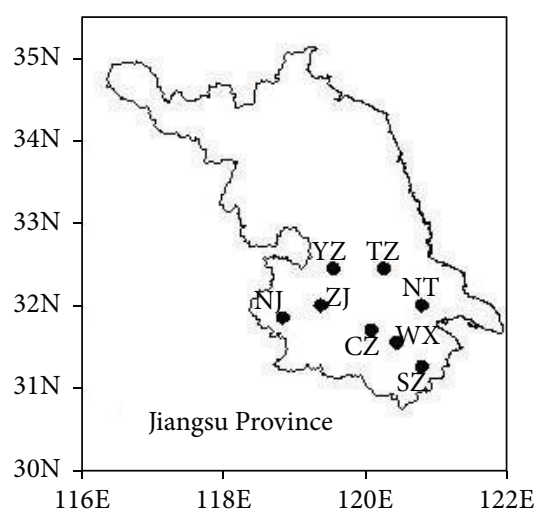

(b)

Figure 1: China (a) and Jiangsu (b). NanJing (NJ), YangZhou (YZ), TaiZhou (TZ), ZhenJiang (ZJ), ChangZhou (CZ), WuXi (WX), SuZhou (SZ), and NanTong (NT).

materials cannot easily disperse; thus, pollutants accumulate. Wu et al. [45] found that vertically dynamic and thermal variations in the boundary layer affect pollutant concentrations. Xiu et al. [46] indicated that stable air over Beijing prevents air movement; as a result, photochemical pollution occurs. Moreover, many experts suggest that a stable temperature inversion, weak atmospheric turbulence, and low winds are the main causes of haze [47-54]. Research has also noted that the diffusion and sedimentation processes of atmospheric chemical materials largely depend on the physical conditions of the vertical boundary layer [55-59].

Existing research focuses on spatiotemporal haze distributions or the synoptic situation and boundary-layer characteristics of specific case studies. However, the weather and boundary-layer climate over many years are not wellknown, particularly in terms of persistent haze and heavy haze. In this study, we classify haze and associated weather systems in southern Jiangsu from 1980 to 2012, especially the surface weather conditions of the persistent regional haze and regional heavy haze. Then, we focus on the boundarylayer characteristics of regional haze events especially the air temperature various in vertical and regional heavy haze in southern Jiangsu. This research provides a basis for haze forecasting.

\section{Data and Methods}

In this study, we obtain data on artificial observation visibility, relative humidity, daily precipitation, and weather in the following cities (Figure 1): NanJing (NJ), YangZhou (YZ), TaiZhou (TZ), ChangZhou (CZ), WuXi (WX), SuZhou (SZ), and NanTong (NT); the data are valid from 1980 to 2012 three times per day $(08 \mathrm{~h}, 14 \mathrm{~h}$, and $20 \mathrm{~h})$. We also use sounding data, such as temperature profiles, for XuZhou (XZ), SheYang (SY), NanJing (NJ), and ShangHai (SH). The sea level pressure at 709 stations across the country from 1980 to 2012 is also used.

We adopt the haze definition of Guo et al. [60]. When the visibility was less than $10 \mathrm{~km}$ and the relative humidity was less than $90 \%$ at any of the three times, the day was considered a haze day unless there was rain, sand storms, blowing sand, suspended dust, smoke, blowing snow, or a snow storm at the same time.

A regional haze event occurred when 3 or more of the 8 cities in southern Jiangsu Province experienced simultaneous haze days. Persistent regional haze was described as regional haze occurrence for 5 or more consecutive days.

Four haze classifications are provided according to visibility (China Meteorological Administration, 2010) [61]. When the visibility is between $5 \mathrm{~km}$ and $10 \mathrm{~km}$, slight haze occurs; when the visibility is between $3 \mathrm{~km}$ and $5 \mathrm{~km}$, mild haze occurs; when the visibility is between $2 \mathrm{~km}$ and $3 \mathrm{~km}$, moderate haze occurs; and when the visibility is less than $2 \mathrm{~km}$, heavy haze occurs. A more severe haze classification is more harmful to humans and is unfavorable for outdoor activities. Therefore, regional heavy haze is defined as when 3 or more of the 8 cities have simultaneous heavy haze.

The lower height of a temperature inversion (LHTI) is the pressure nearest the surface when the first temperature inversion layer develops. The upper height of a temperature inversion (UHTI) is the pressure at the top of the first temperature inversion layer. The temperature difference in the temperature inversion is the upper temperature of the first temperature inversion layer (UTTI) minus the low-level temperature of the first temperature inversion layer (LTTI). The height difference in the temperature inversion is the pressure of the LHTI minus the pressure of the UHTI. The intensity of the temperature inversion (ITI) is the temperature difference divided by the height difference multiplied by $100\left({ }^{\circ} \mathrm{C} / 100 \mathrm{hPa}\right)$ :

$$
\mathrm{ITI}=\left(\frac{(\mathrm{UTTI}-\mathrm{LTTI})}{(\mathrm{LHTI}-\mathrm{UHTI})}\right) * 100 .
$$

\section{Results}

3.1. Synoptic Conditions of Regional Haze. According to the definition of regional haze, 4851 regional haze days occurred 


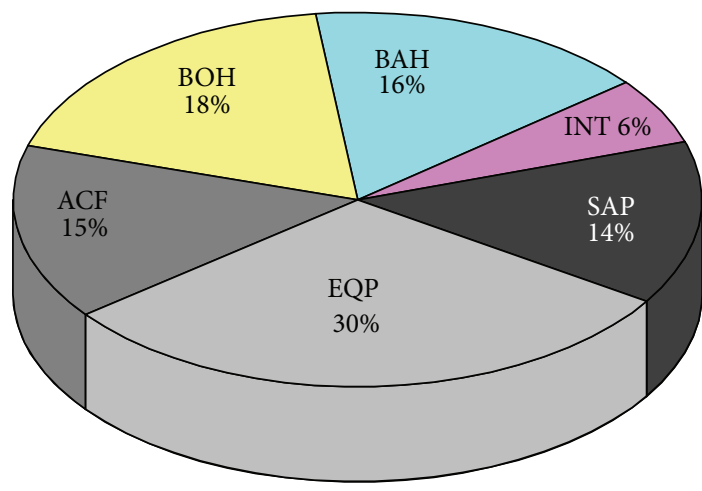

Figure 2: Surface conditions of regional haze days (equalized pressure: EQP, advancing edge of a cold front: ACF, base of high pressure: $\mathrm{BOH}$, backside of high pressure: $\mathrm{BAH}$, inverted trough: INT, and saddle pressure: SAP).

from 1980 to 2012. Regional haze lasted for 5 or more days 246 times.

3.1.1. Surface Conditions of Regional Haze. According to the definition of regional haze, we analyze the sea level pressure. Six types of weather patterns are considered: equalized pressure (EQP), advancing edge of a cold front (ACF), base of high pressure $(\mathrm{BOH})$, backside of high pressure $(\mathrm{BAH})$, inverted trough (INT), and saddle pressure (SAP). Figure 2 shows the frequency of the pressure types. When regional haze occurs, the main surface conditions (79\% of cases) are EQP, ACF, BOH, or BAH.

Figure 3(a) shows the 246 instances of 5 or more consecutive regional haze days. In this figure, there are 97 cases of 5 days, 51 cases of 6 days, 32 cases of 7 days, 21 cases of 8 days, 16 cases of 9 days, 11 cases of 10 days, and 18 cases of 10 or more days. The longest haze case was 18 days; however, $80 \%$ of the haze cases were 8 days or less.

Figure 3(b) shows the surface conditions of 5 or more consecutive regional haze days from 1980 to 2012. Compared with Figure 2, the main structures are similar. The primary weather pattern was EQP (36\% of the cases). $\mathrm{BOH}$ and ACF accounted for $19 \%$ and $17 \%$, respectively, of the cases; $\mathrm{BAH}$ occurred during $12 \%$ of the haze events. The 4 pressure types accounted for $84 \%$ of the cases overall.

By filtering the regional haze days, we obtained 50 days with regional heavy haze.

The surface conditions of the regional heavy haze were mainly equalized pressure (EQP), advancing edge of a cold front $(\mathrm{ACF})$, base of high pressure $(\mathrm{BOH})$, backside of high pressure (BAH), and inverted trough (INT). (1) EQP (Figure 4(a)): when the cold air was blocked in the north, the domain was controlled by equalized pressure. (2) ACF (Figure 4(b)): when the cold air strongly advanced, the domain was controlled by the advancing edge of the cold front. (3) BOH (Figure 4(c)): when the mainland high pressure moved southward, the domain was overtaken by the base of the high pressure. (4) BAH (Figure 4(d)): when the mainland high pressure disbanded, the domain was located on the backside of the high pressure. (5) INT (Figure 4(e)): when the domain was controlled by the back of the weak high pressure, the high pressure receded, the inverted trough developed, and the domain was overtaken by the top of the inverted trough.

3.1.2. Surface Weather Conditions of Persistent Regional Haze. We analyze the surface conditions during the 246 regional persistent haze cases. Four types of persistent regional haze can be obtained according to the surface weather conditions on the first day of the regional haze event (Figure 4): EQP, $\mathrm{ACF}, \mathrm{BOH}$, and $\mathrm{BAH}$. These 4 patterns occurred 214 times. In 5 instances, the 4 patterns were hybrids during regional haze events; the remaining weather patterns occurred 27 times. The detailed variations of the 4-type surface weather conditions are presented as below.

Type I (EQP) occurred 91 times (Figure 5). Often, the domain experienced $\mathrm{EQP}$, then $\mathrm{BOH}, \mathrm{BAH}$, or $\mathrm{ACF}$. If the EQP transitioned to $\mathrm{BOH}$, along with high-pressure movement toward the sea, then the region was controlled by $\mathrm{BAH}$, cold air pushed southward, and the domain was controlled by the ACF (9 instances). If the EQP transitioned to $\mathrm{BAH}$, then there was a low trough developing in the southwest. Then, the domain was controlled by the top of an inverted trough. If the EQP transitioned to an ACF, then the domain would be controlled by the BAH when the high pressure weakened. Following the high-pressure progression toward the sea, the area was controlled by SAP. At this time, there was a cold vortex blocking the cold air to the north, and the domain was controlled by the EQP again. If the domain was controlled by the EQP on the first day of a persistent haze case, then an inverted trough developed to the southwest and moved toward the northeast; the domain was controlled by the top of the inverted trough. Because the inverted trough moved out of the domain, the high pressure over the mainland shifted to the southeast; if the speed was slow, then the domain was controlled by the EQP. Or when the speed was rapid, the domain was controlled by the BAH. The domain was controlled by the EQP 70 times at the beginning of a haze period and by $\mathrm{BOH}, \mathrm{BAH}$, or $\mathrm{ACF}$ at the end of the period.

Type II (ACF) occurred 57 times (Figure 5). At the beginning of persistent haze cases, the domain was controlled by an ACF. When the cold front passed, the domain was controlled by the EQP. Notably, two cold air processes occurred during haze cases. There were three options during the two cold-front processes. First, when the high pressure was separated into two small high-pressure systems, the domain was occupied by the BAH and then by the SAP. When another cold air system moved southward, the domain was controlled by the ACF (14 instances). Second, if there was a vortex over North China, when the vortex moved toward the sea and another cold air system moved southward, the domain was dominated by the ACF (21 instances). Third, if the vortex over North China was strong and weakened the cold air, when the vortex moved seaward, the domain was occupied by BAH and then by the ACF ( 8 instances). Fourth, when the high pressure was undisturbed or the cold vortex was not blocked, the domain was controlled by the $\mathrm{BOH}$, 


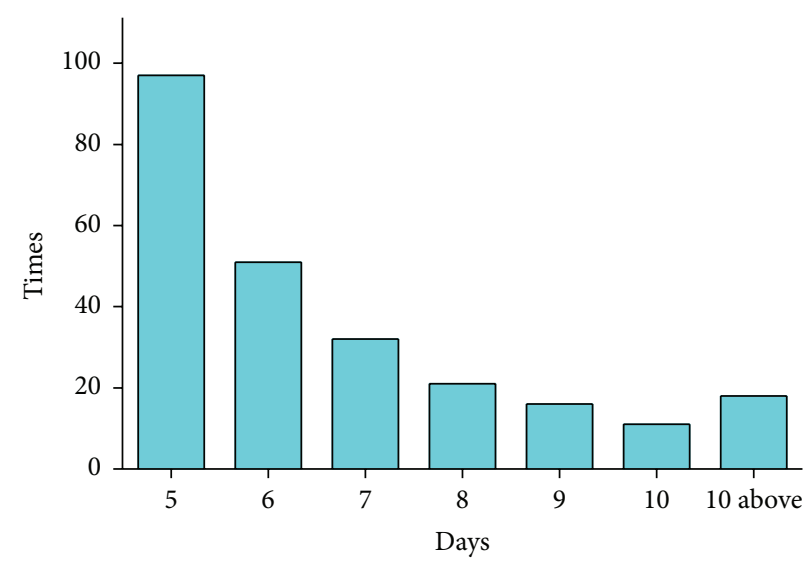

(a)

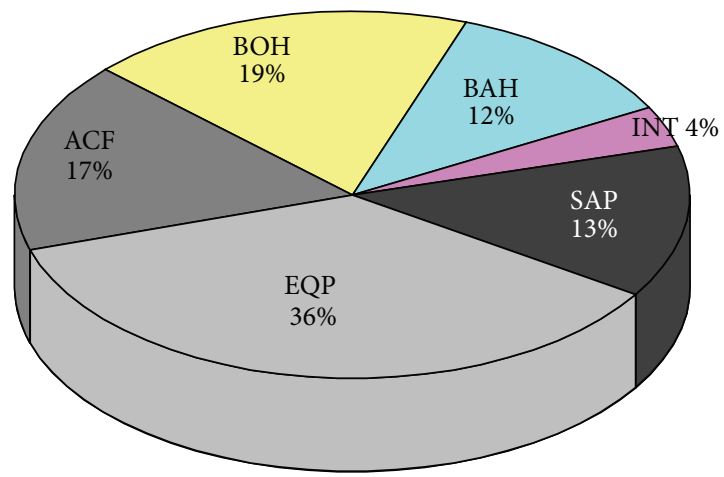

(b)

FIGURE 3: Count of consecutive regional haze days between 1980 and 2012 (a); surface conditions during 5 or more consecutive haze days (b) (equalized pressure: EQP, advancing edge of a cold front: ACF, base of high pressure: $\mathrm{BOH}$, backside of high pressure: $\mathrm{BAH}$, inverted trough: INT, and saddle pressure: SAP).

the EQP of high pressure, and then cold air. Overall, type II is characterized by two passages of cold air. The greatest differences were the high-pressure dissipation, cold vortex blocking, low-pressure vortex movement toward sea, and the time interval between the two cold air systems.

Type III (BOH), which is complex, occurred 54 times (Figure 5). When the haze days began, the domain was characterized by the $\mathrm{BOH}$.

When the system was moving slowly, it was controlled by the EQP, which is similar to type I. If there was an inverted trough developing, then the top of the trough became dominant. If cold air moved southward, then the domain was controlled by the ACF and then by the EQP. Following the southward trajectory of the high pressure, the area was occupied by $\mathrm{BOH}$. However, when the oceanic high pressure and vortex in the north occurred at the same time, SAP was present over the domain; then, the ACF was dominant when cold air pushed south.

In that case, if the system moved quickly, then the domain was controlled by the BAH as the high pressure moved out. At that time, if an inverted trough was developing, then it dispersed the cold air and the top of the inverted trough was dominant. Then, the ACF accompanying the cold air strengthened. On condition that the cold vortex in the north was replaced, the SAP and EQP were dominant, followed by the top of the inverted trough. Type III was characterized by an inverted low-pressure trough and saddle pressure due to the weak high pressure or cold vortex in the northeast or the vortex in the southwest.

Type IV (BAH) occurred 12 times (Figure 5). When a haze case began, the domain was controlled by the BAH. If the cold vortex was weak when the cold air strongly pushed toward the south, then the domain was occupied by the ACF (4 instances). In case the cold vortex was strong and steady and accompanied by mainland high pressure to the east, then the domain was controlled by the high EQP. Then, the cold vortex strengthened in the north, and the high pressure moved out; the domain was controlled by the $\mathrm{BOH}$. Then, the EQP dominated, with high pressure moving toward the sea and the cold vortex moving eastward. At that time, a cold-core high-pressure system developed; the domain was controlled by the BOH. Type IV was characterized by a cold vortex. The differences stemmed from the strength of the cold vortex in the northeast and the speed or strength of the cold air movement.

\section{Hybrid patterns occurred 5 times.}

Between 1994-10-30 and 1994-11-12, when the haze began, the domain was controlled by the ACF and then the EQP; however, because of the effect of the cold vortex in the north, the high pressure was broken up and the domain was overtaken by the BAH. As the cold vortex moved south, the domain was located on the backside of the cold vortex. Following cold air intrusion and the cold vortex retreat to the north and east, the domain experienced the $\mathrm{BOH}$. Then, the cold air in the north developed again, and the domain was overtaken by the $\mathrm{BAH}$ and then the SAP as the high pressure dissipated. At that time, there was weak cold air to the south; the domain was occupied by the EQP of high pressure and then by $\mathrm{BOH}$. When the high pressure moved out, the $\mathrm{BAH}$ dominated. Then, the high pressure moved toward the sea, and the domain was again controlled by the EQP. When the newly formed cold air mass moved south, an inverted trough developed in the southwest and moved northeast; then, the domain was located along the top of the inverted trough. This entire process characterized the ACF transition into the $\mathrm{BOH}$. The haze ended at the base of the high pressure with strong cold air.

Between 2004-11-02 and 2004-11-08, when the haze started, the domain experienced the EQP. Following the high-pressure movement, the domain was controlled by the $\mathrm{BAH}$. Along with the advancing cold air, the $\mathrm{BOH}$ and $\mathrm{BAH}$ replaced the high pressure. At that time, there was inverted trough in the southwest, which tracked toward the northeast; finally, the domain was centered on the inverted trough. This process characterized the EQP transition into the $\mathrm{BOH}$. 


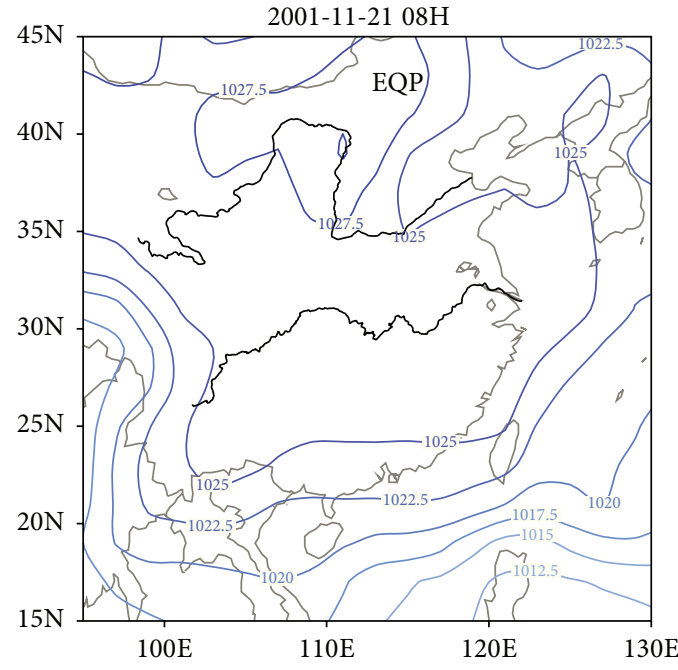

(a)

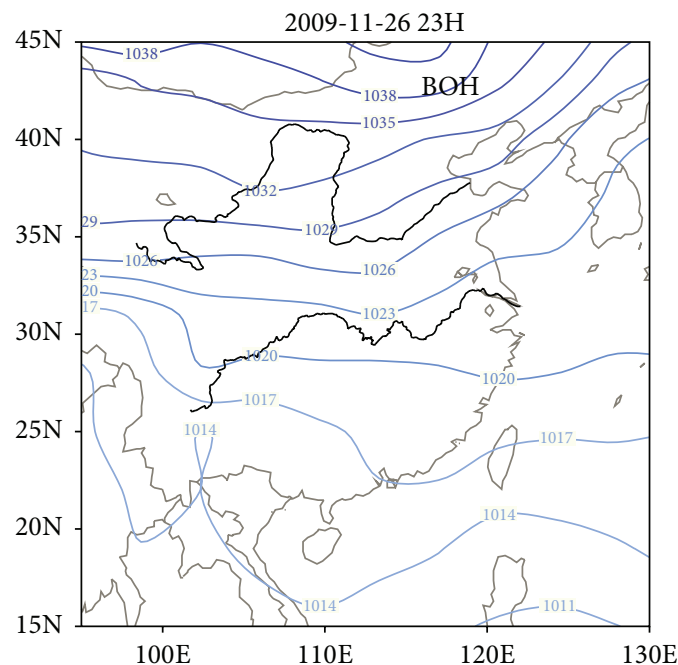

(c)

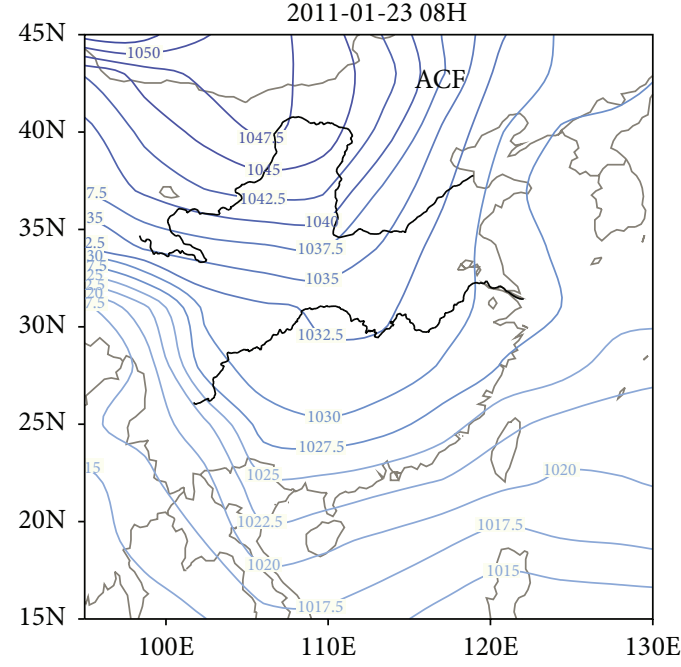

(b)

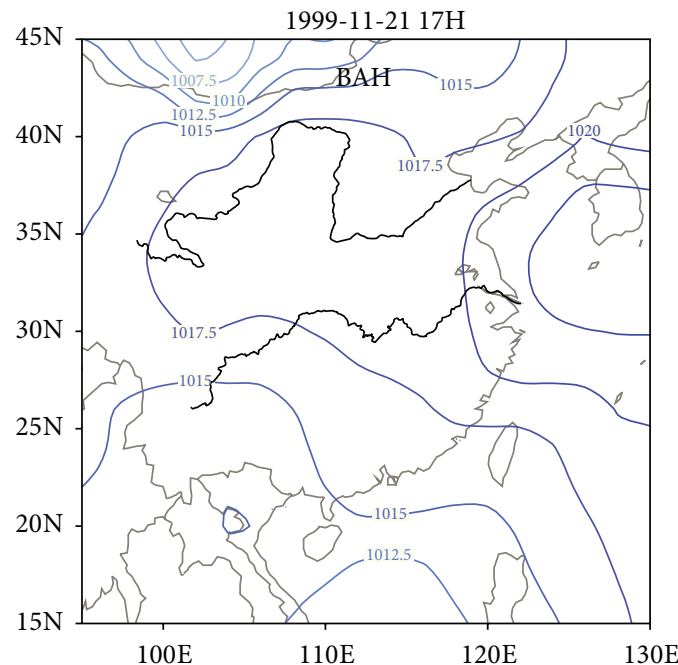

(d)

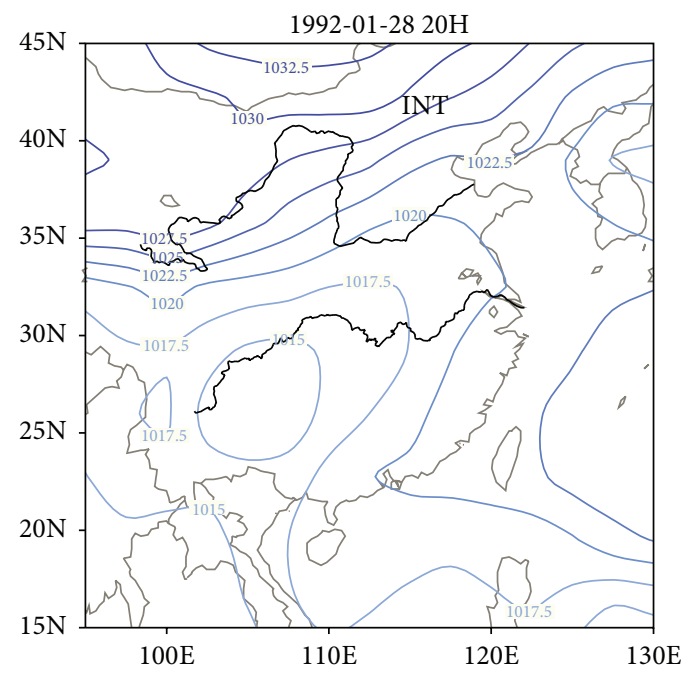

(e)

FIGURE 4: Surface conditions during heavy haze days ((a) equalized pressure: EQP, (b) advancing edge of a cold front: ACF, (c) base of high pressure: $\mathrm{BOH},(\mathrm{d})$ backside of high pressure: $\mathrm{BAH}$, and (e) inverted trough: INT). 


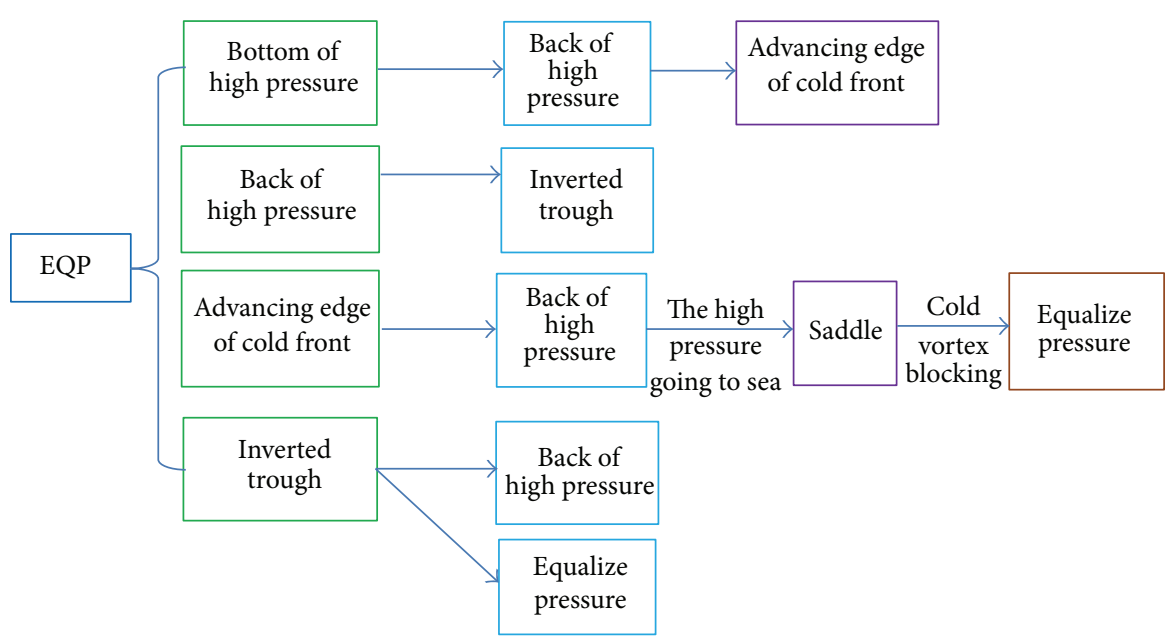

(I)

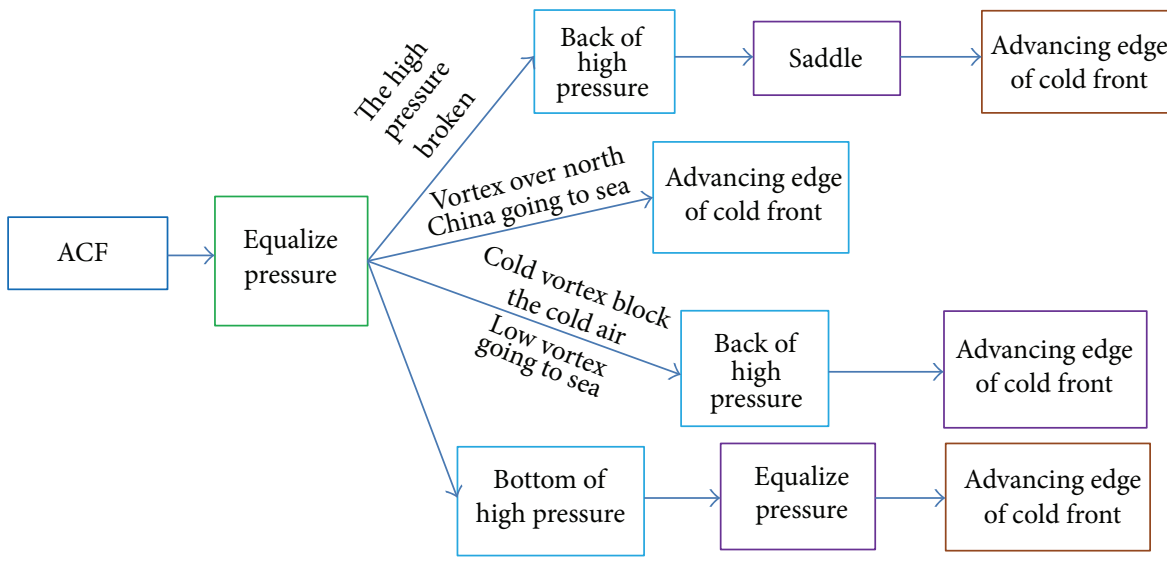

(II)

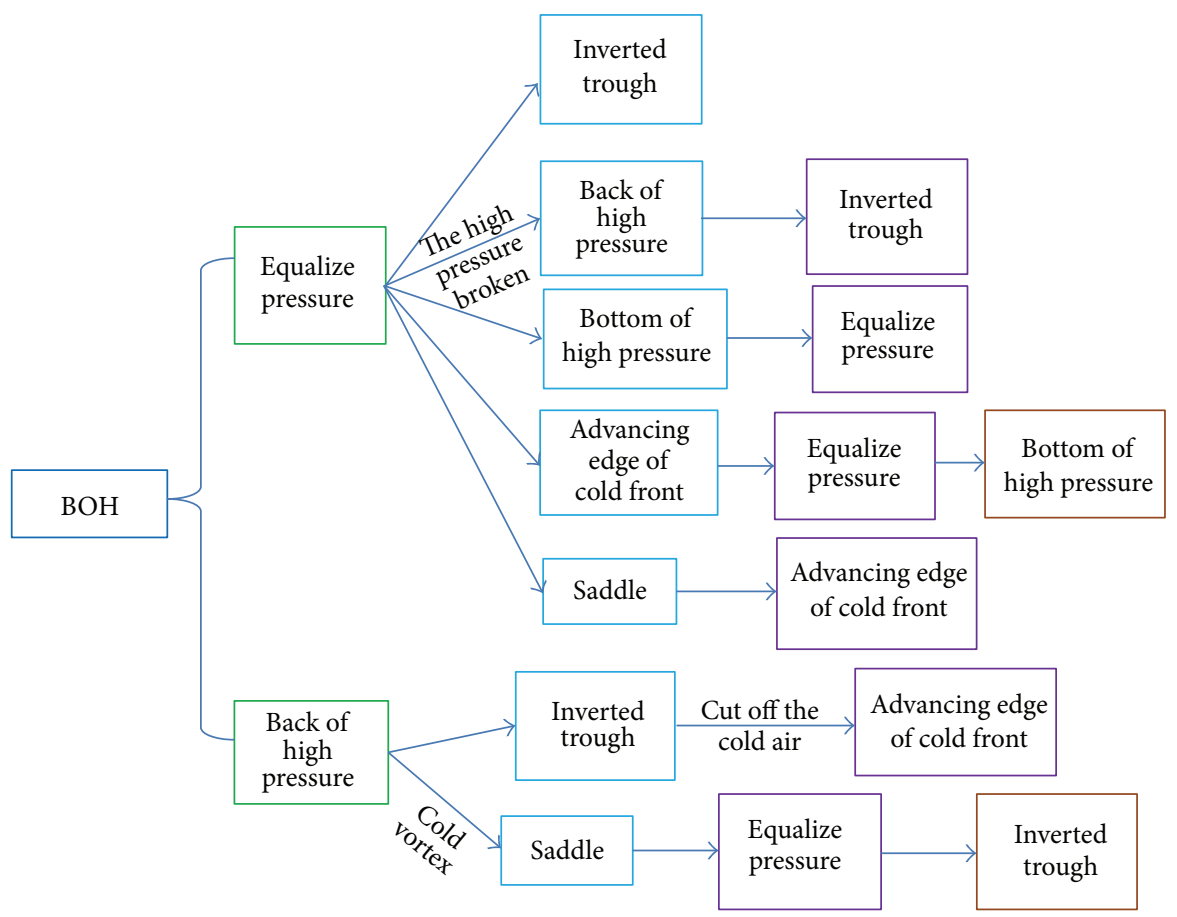

(III)

Figure 5: Continued. 


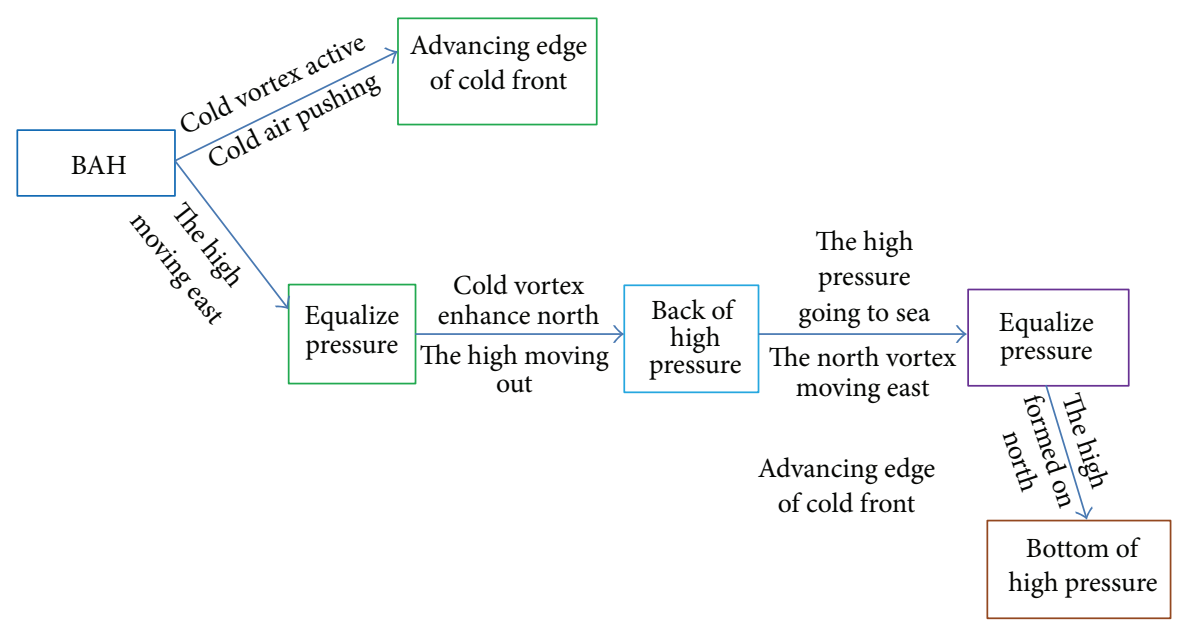

(IV)

FIGURE 5: The 4 types of weather patterns during regional persistent haze events (equalized pressure: EQP, advancing edge of a cold front: ACF, base of high pressure: $\mathrm{BOH}$, backside of high pressure: $\mathrm{BAH}$, inverted trough: INT, and saddle pressure: SAP).

From 2006-03-01 to 2006-03-08 and 2009-11-20 to 2009$11-26$, these two synoptic patterns were the same. Initially, the domain was located along the ACF as the cold air passed. Then, the domain was controlled by the EQP of the high pressure and successively by the $\mathrm{BAH}$ as the high pressure moved toward the sea. Then, the domain was dominated by the EQP. As the cold air gradually moved east, the BAH dominated. These two processes describe the ACF transition to the BAH.

Between 2011-03-08 and 2011-03-13, when the haze began, the domain was located along the ACF. As the cold air advanced, the EQP of the high pressure dominated. When the high pressure moved east, the domain was located on the $\mathrm{BAH}$. At that time, strong cold air pushed south, but the low inverted trough weakened the cold air mass, so the domain was located at the top of the inverted trough. The haze ended with another cold air intrusion. This process describes the $\mathrm{ACF}$ transition to the $\mathrm{BOH}$.

The other weather patterns occurred 27 times; 23 instances between May to September were characterized by a northern cold vortex shaping the structure of the northerly low and southerly high, the base of the low vortex, or typhoons. Four instances occurred in autumn and winter. For example, from 1990-12-04 to 1990-12-10, 1998-04-14 to 199804-21, and 1998-11-11 to 1998-11-15, the domain was affected by the base of the cold vortex from the north. As the cold vortex moved toward the sea, the domain experienced the EQP. Then, the domain experienced the ACF because the cold air moved south.

Between 2002-10-08 and 2002-10-16, after the BOH, the domain was affected by BAH as high pressure moved toward the sea. Then, the domain was controlled by the EQP. At that time, another mainland high-pressure system was located to the south: that is, the $\mathrm{BOH}$ transitioned to the EQP. Then, the low vortex of the north was affected by the base of the low vortex. As the low vortex moved toward the east and the sea, the domain was controlled by the backside of the high pressure over the sea.
3.1.3. Surface Conditions of Regional Heavy Haze. Table 1 shows the categories of the surface and high-pressure patterns during regional heavy haze. When regional heavy haze occurred, the $850 \mathrm{hPa}$ pressure was uniform near the backside of the high-pressure system or the saddle pressure. At $500 \mathrm{hPa}$, the airflow was northwesterly, westerly, or southwesterly.

Table 2 reveals the seasonal distribution of regional heavy haze during different pressure types. From the table, the EQP and the ACF occurred 12 times, the $\mathrm{BOH}$ occurred 5 times, the BAH occurred 10 times, the INT occurred 6 times, and other weather types occurred 5 times. The main 4 types accounted for $78 \%$ of the haze cases. We also find that the regional heavy haze mainly occurred in autumn and winter. Only 3 events occurred in summer.

\subsection{Boundary-Layer Structures of Regional Haze}

3.2.1. Boundary-Layer Structures of Persistent Regional Haze. According to the previous analysis, we selected representative regional haze events during $\mathrm{EQP}, \mathrm{ACF}, \mathrm{BOH}$, and $\mathrm{BAH}$.

(1) EQP. On the basis of the EQP departure, we selected 5 typical events (Table 3 ). Then, we analyzed the 5 associated processes.

Figure 6 shows the temperature profiles of the events. In Figure 6 , the $x$-axes are the data and the $y$-axes are the pressures. The shaded area is the vertical temperature distribution. The broken lines are the LHTI and UHTI of the temperature inversion. The dotted line is the ITI of the temperature inversion (the same below).

From 1984-10-31 to 1984-11-08, when the haze days began, the domain had uniform pressure ahead of the cold front; then, cold air advanced and the domain was controlled by the ACF. When the high pressure weakened, the domain was under the BAH. Then, the high pressure moved seaward, and the domain was controlled by the SAP. However, at that time, the cold vortex to the north blocked the cold air, and 
TABLE 1: Surface and high-pressure conditions on heavy haze days.

\begin{tabular}{|c|c|c|c|c|c|c|}
\hline Series & Types & Date (yyyy-mm-dd) & $500 \mathrm{hPa}$ & $850 \mathrm{hPa}$ & $\begin{array}{l}\text { Stations recording } \\
\text { heavy haze days }\end{array}$ & $\begin{array}{c}\text { Stations recording } \\
\text { haze days }\end{array}$ \\
\hline 1 & \multirow{12}{*}{ EQP } & $1987-12-26$ & Southwest & Equalizing zone & 4 & 7 \\
\hline 2 & & $1992-10-28$ & West & Equalizing zone & 3 & 6 \\
\hline 3 & & 1994-04-28 & Northwest & Equalizing zone & 3 & 7 \\
\hline 4 & & $1998-12-27$ & Northwest to west & Equalizing zone & 3 & 7 \\
\hline 5 & & $2001-11-21$ & West & Equalizing zone & 3 & 8 \\
\hline 6 & & $2001-11-22$ & West & Equalizing zone & 3 & 7 \\
\hline 7 & & 2002-02-05 & Northwest to west & Equalizing zone & 3 & 7 \\
\hline 8 & & $2005-10-26$ & Northwest to southwest & Saddle & 3 & 8 \\
\hline 9 & & 2007-04-06 & West & Equalizing zone & 3 & 8 \\
\hline 10 & & $2008-07-17$ & Equalizing zone & Saddle & 3 & 5 \\
\hline 11 & & 2008-11-01 & West & Equalizing zone & 3 & 6 \\
\hline 12 & & 2012-03-06 & Southwest to west & & 3 & 8 \\
\hline 13 & \multirow{12}{*}{$\mathrm{ACF}$} & $1981-12-28$ & West & Saddle & 3 & 8 \\
\hline 14 & & $1985-11-16$ & West & Equalizing zone & 3 & 8 \\
\hline 15 & & $1990-10-15$ & Northwest & Equalizing zone & 3 & 8 \\
\hline 16 & & $1999-10-28$ & West & & 3 & 8 \\
\hline 17 & & $2002-12-31$ & Northwest & Inverted trough & 3 & 8 \\
\hline 18 & & $2003-12-12$ & Northwest & Equalizing zone & 3 & 6 \\
\hline 19 & & $2003-12-16$ & Northwest & Base of cold vortex & 3 & 7 \\
\hline 20 & & $2003-12-17$ & Northwest & Base of cold vortex & 3 & 8 \\
\hline 21 & & 2009-01-09 & Northwest to west & & 3 & 8 \\
\hline 22 & & $2010-11-21$ & Front of trough & Base of trough & 3 & 7 \\
\hline 23 & & 2011-01-23 & Northwest to west & Equalizing zone & 3 & 5 \\
\hline 24 & & $2012-01-20$ & West & Equalizing zone & 3 & 8 \\
\hline 25 & \multirow{5}{*}{$\mathrm{BOH}$} & 1992-01-25 & West & Equalizing zone & 3 & 8 \\
\hline 26 & & 2005-10-18 & West & Equalizing zone & 3 & 8 \\
\hline 27 & & 2008-03-11 & West & Backside of the high pressure & 3 & 8 \\
\hline 28 & & $2009-11-26$ & West & Backside of the high pressure & 3 & 6 \\
\hline 29 & & 2011-10-08 & West & Backside of the high pressure & 3 & 6 \\
\hline 30 & \multirow{10}{*}{$\mathrm{BAH}$} & $1987-12-24$ & West & Equalizing zone & 3 & 6 \\
\hline 31 & & $1992-01-27$ & Northwest & Equalizing zone & 4 & 8 \\
\hline 32 & & 1992-05-31 & Equalizing zone & Equalizing zone & 3 & 6 \\
\hline 33 & & $1992-11-14$ & & & 3 & 6 \\
\hline 34 & & 1996-04-26 & Northwest & & 3 & 8 \\
\hline 35 & & $1999-11-21$ & West & & 3 & 8 \\
\hline 36 & & $2005-10-31$ & West & Equalizing zone & 3 & 6 \\
\hline 37 & & 2008-04-18 & Southwest & Backside of the high pressure & 3 & 6 \\
\hline 38 & & $2008-10-15$ & Southwest to west & Equalizing zone & 3 & 8 \\
\hline 39 & & 2011-06-13 & West & Equalizing zone & 4 & 6 \\
\hline 40 & \multirow{6}{*}{ INT } & 1984-04-26 & Northwest to southwest & Equalizing zone & 3 & 7 \\
\hline 41 & & $1992-01-28$ & Northwest & Equalizing zone & 3 & 8 \\
\hline 42 & & 1994-11-11 & Northwest to southwest & Equalizing zone & 3 & 6 \\
\hline 43 & & 2004-02-19 & Front of ridge & Backside of the high pressure & 3 & 8 \\
\hline 44 & & 2004-05-10 & Front of ridge & Backside of the high pressure & 3 & 8 \\
\hline 45 & & 2009-11-08 & Southwest to west & Saddle & 3 & 7 \\
\hline 46 & \multirow{5}{*}{ Others } & $2002-01-03$ & Northwest & & 3 & 5 \\
\hline 47 & & $2003-06-25$ & Equalizing zone & Saddle & 3 & 8 \\
\hline 48 & & 2004-11-07 & West & Equalizing zone & 4 & 8 \\
\hline 49 & & $2008-05-26$ & Northwest & Equalizing zone & 4 & 8 \\
\hline 50 & & 2011-06-03 & Equalizing zone & Equalizing zone & 3 & 7 \\
\hline
\end{tabular}

Equalized pressure: EQP, advancing edge of a cold front: ACF, base of high pressure: BOH, backside of high pressure: BAH, and inverted trough: INT. 
TABLE 2: Types of consecutive heavy haze days.

\begin{tabular}{lccccc}
\hline Type & & \multicolumn{5}{c}{ Month } \\
& $3-5$ & $6-8$ & $9-11$ & $12-2$ & $1-12$ \\
\hline EQP & 3 & 1 & 5 & 3 & 12 \\
ACF & & & 4 & 8 & 12 \\
BOH & 1 & & 3 & 1 & 5 \\
BAH & 3 & 1 & 4 & 2 & 10 \\
INT & 2 & & 2 & 2 & 6 \\
Others & 2 & 1 & 1 & 1 & 5 \\
\hline
\end{tabular}

Equalized pressure: EQP, advancing edge of a cold front: ACF, base of high pressure: $\mathrm{BOH}$, backside of high pressure: $\mathrm{BAH}$, and inverted trough: INT.

TABLE 3: Typical processes of equalized pressure (EQP) during consecutive regional haze days.

\begin{tabular}{lccc}
\hline Series & Start day & End day & Duration \\
\hline 1 & $1984-10-31$ & $1984-11-08$ & 9 \\
2 & $1991-06-20$ & $1991-06-27$ & 8 \\
3 & $1999-09-25$ & $1999-09-29$ & 5 \\
4 & $2000-12-03$ & $2000-12-09$ & 7 \\
5 & $2007-09-08$ & $2007-09-17$ & 10 \\
\hline
\end{tabular}

the domain was again controlled by the EQP. When the haze began, the temperature inversion layer extended from the surface to $950 \mathrm{hPa}$. During the haze days, the base of the temperature inversion remained, and the temperature inversion at $950 \mathrm{hPa}$ developed until the end of the haze period. The intensity of the temperature inversion had an obvious diurnal variation: strong in the daytime and weak in the nighttime. Meanwhile, the temperature inversion was weak in the mid-haze period. During this process, the temperature inversion layer was near the surface and shallow; double temperature inversion layers occurred at low altitudes.

From 1991-06-20 to 1991-06-27, the temperature inversion occurred in the middle of the persistent haze case. The inversion was low, thin, and weak. During this process, the summer harvest and a large seeded area occupied the domain. The weather was hot and humid, in accordance with the Meiyu period. Therefore, we assumed that the haze was mainly affected by solid particles and gas produced by straw burning [62, 63]; the particles and gas accumulated with the high humidity, causing persistent haze.

From 1999-09-25 to 1999-09-29, when the haze days began, the domain had uniform high pressure. Then, the high pressure moved eastward, and the domain was on the backside of the high pressure. At that time, the inverted trough was developing, and the domain was located along the top of the trough. When the newly formed cold air mass moved southward, the domain was affected by the ACF. When the haze began, the temperature inversion was just above the surface. The event lasted 2 days; 3 stations (XZ, SH, and SY) had high-altitude temperature inversions, but the inversion at the NJ station was still near the surface. In Figure 6(c), the surface-layer temperature inversion was thin at the NJ station, and high-altitude inversions at the other stations were also thin. The variable temperature inversion intensity was severe.
TABLE 4: Typical processes of the advancing edge of a cold front (ACF) pattern during persistent regional haze days.

\begin{tabular}{lccc}
\hline Series & Start day & End day & Duration \\
\hline 1 & $1982-12-11$ & $1982-12-24$ & 14 \\
2 & $2003-10-24$ & $2003-11-03$ & 11 \\
3 & $2005-01-11$ & $2005-01-20$ & 10 \\
4 & $2011-12-28$ & $2012-01-07$ & 11 \\
\hline
\end{tabular}

Initially, the inversion intensity was strong to the north and weak to the south. Then, the temperature inversion weakened at $\mathrm{XZ}$ and SY and strengthened at $\mathrm{NJ}$ and SH. At the end of the haze period, the temperature inversion was strong at XZ and weak at the other stations. This difference may be due to the movement of the system during the late haze period.

From 2000-12-03 to 2000-12-09, at the early stage of the haze, the temperature inversion or isothermal layer was recorded at all 4 stations. The height of the layer increased from north to south. When the haze days were consecutive, the surface layer of the temperature inversion was still recorded at XZ, SY, and NJ. However, the significant daily variation at $\mathrm{SH}$ was characterized as low in the daytime and high at nighttime. The temperature inversion was thin, with a variable intensity. When the intensity was strong, the top of the temperature inversion layer was low and vice versa.

From 2007-09-08 to 2007-09-17, the temperature inversion was initially near the surface. With persistent haze days, the base of the temperature inversion minimally changed at $\mathrm{XZ}$ and SY, but the thickness increased. At that time, the base of the temperature inversion clearly lifted, and the thickness increased at $\mathrm{NJ}$ and $\mathrm{SH}$. At the end of the haze period, the base of the temperature inversion gradually lifted, and the thickness weakened from north to south. The intensity was initially stronger to the north. With persistent haze development, the intensity was weaker at XZ and SY and stronger at $\mathrm{NJ}$ and $\mathrm{SH}$ during the middle of the haze period.

Table 3 shows that a haze event between 5 and 10 days has an EQP. The duration depended on the system development at the middle and end of the persistent haze case. The duration was shorter when the domain was controlled by the EQP, the $\mathrm{BAH}$, and then the INT. If the domain was initially controlled by the EQP and then overtaken by the INT, EQP, and SAP, the duration was longer. The base of the shallow temperature inversion layer was low, and the intensity was variable for different cases.

(2) ACF. Based on the developing features of an ACF, we selected 4 significant processes (Table 4). Based on the table, we find that this weather pattern lasts several days. We analyzed the 4 processes (Figure 7).

From 1982-12-11 to 1982-12-24 (Figure 7(a)), when the haze days began, the temperature inversions were elevated or no temperature inversion occurred. Following the hazeday development, the temperature inversion occurred near the ground. The top of the temperature inversion layer was approximately $1000 \mathrm{hPa}$. At the middle and end of the hazeday period, the surface layer of the temperature inversion persisted, and the multiple layers of the temperature inversion 


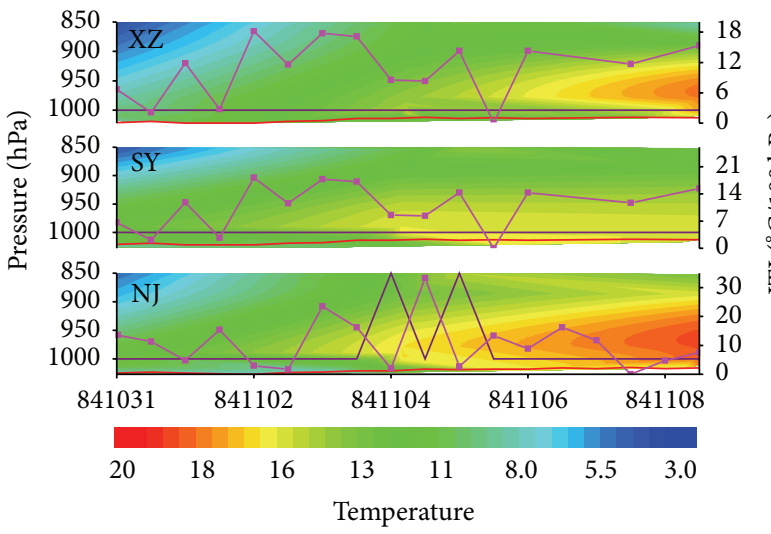

(a)

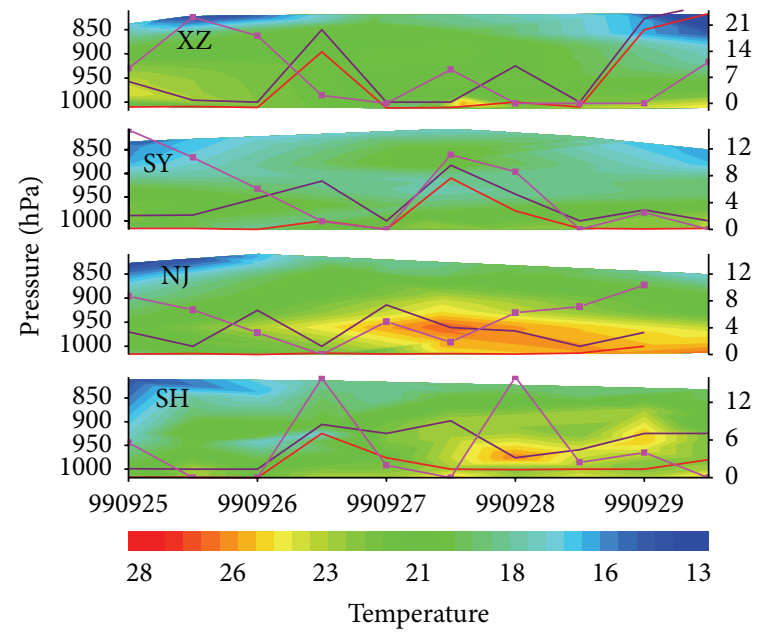

(c)

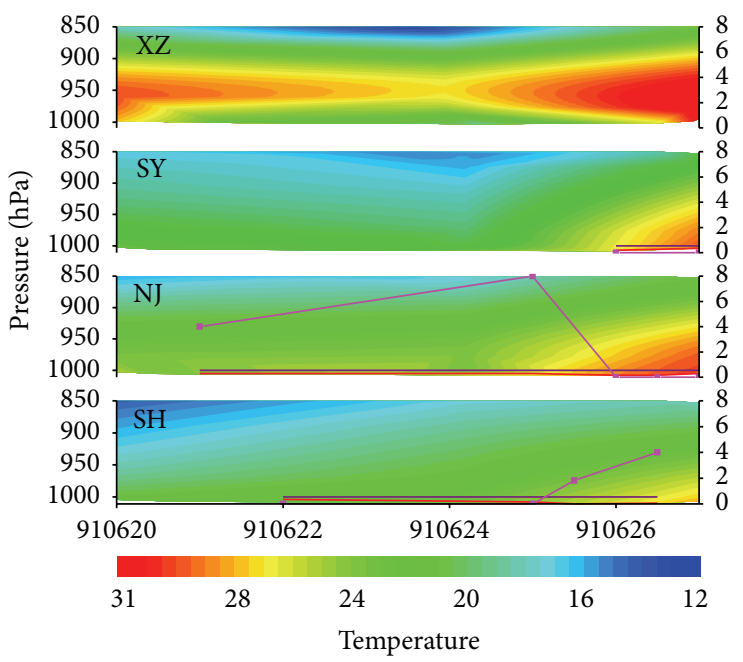

(b)

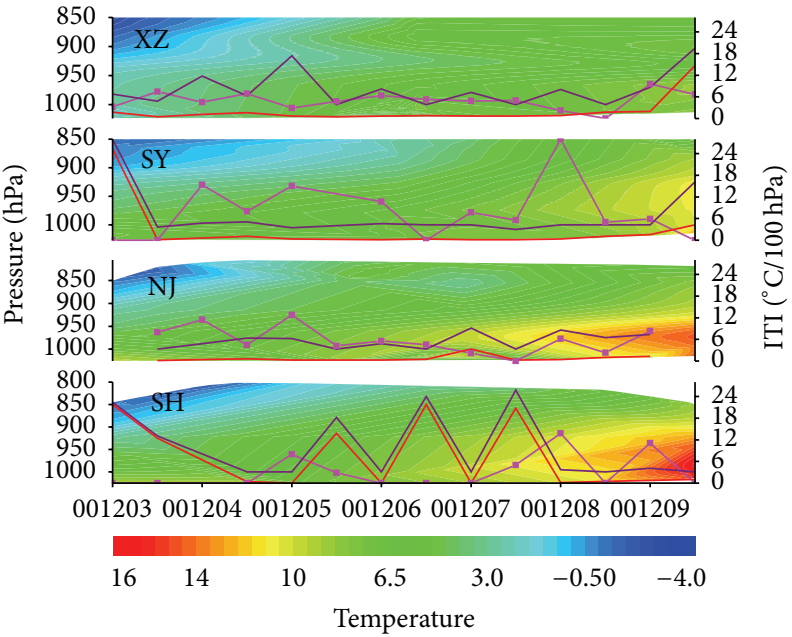

(d)

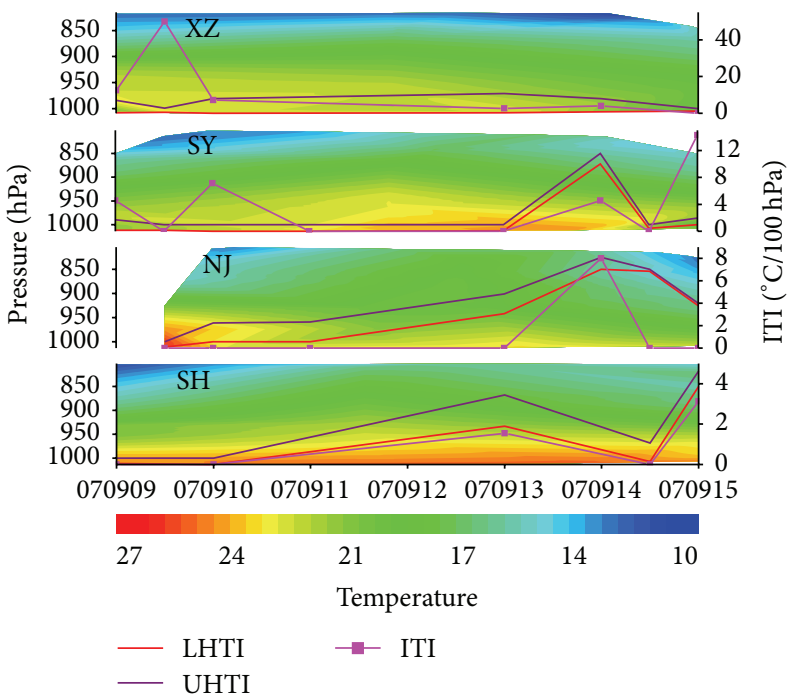

(e)

FIgURE 6: Temperature profiles of the equalized pressure (EQP) type during persistent regional haze days at XuZhou (XZ), SheYang (SY), NanJing (NJ), and ShangHai (SH) ((a) 1984-10-31-1984-11-08, (b) 1991-06-20-1991-06-27, (c) 1999-09-25-1999-09-29, (d) 2000-12-03-200012-09, and (e) 2007-09-08-2007-09-17). Lower height of temperature inversions (LHTI), upper height of temperature inversions (UHTI), and intensities of the temperature inversions (ITI). 


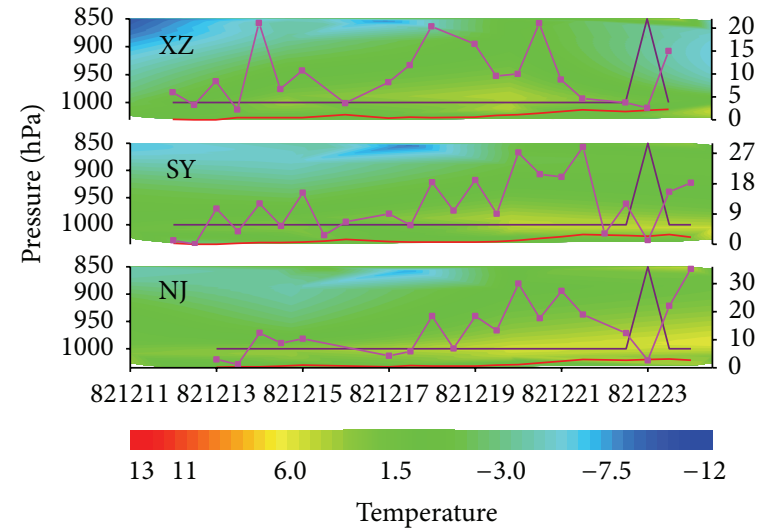

(a)

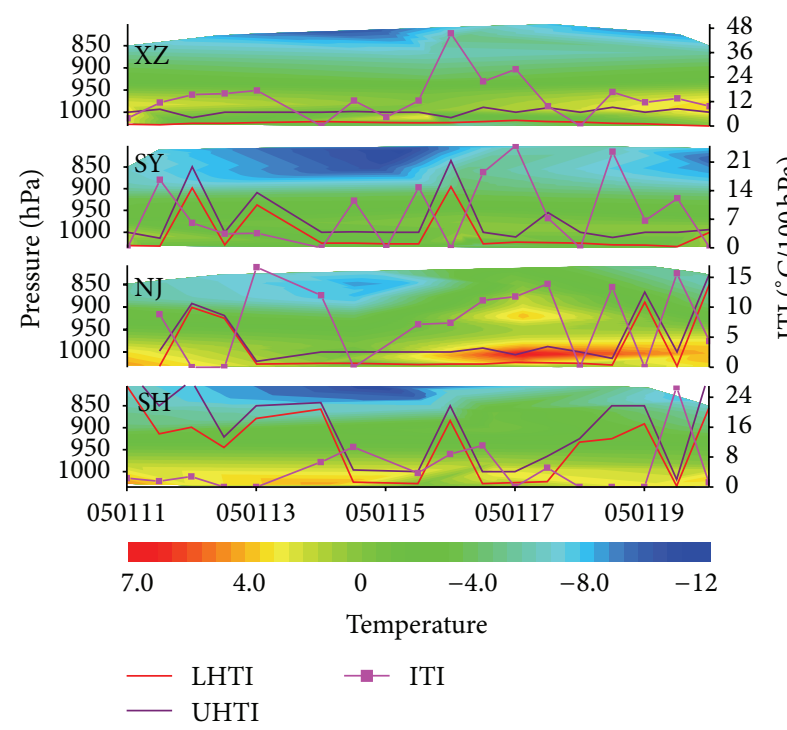

(c)

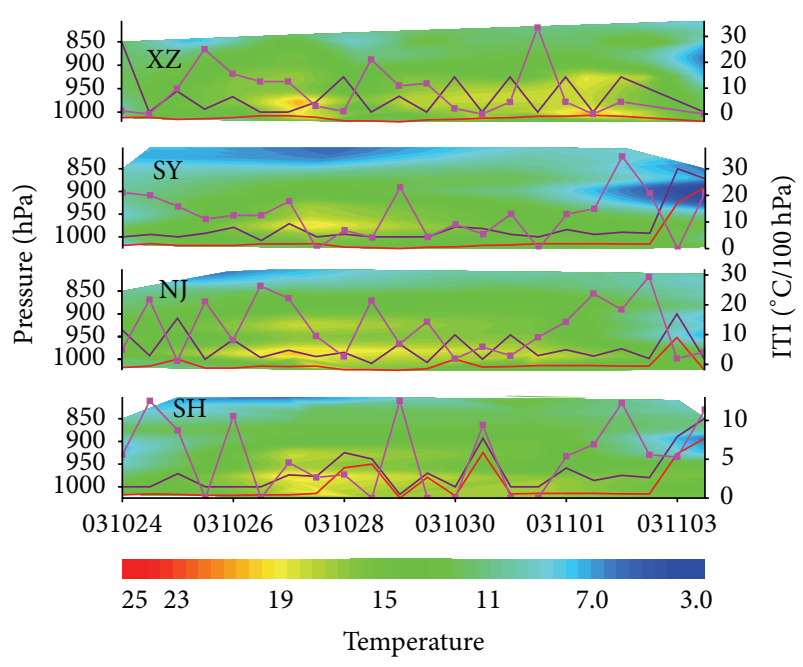

(b)

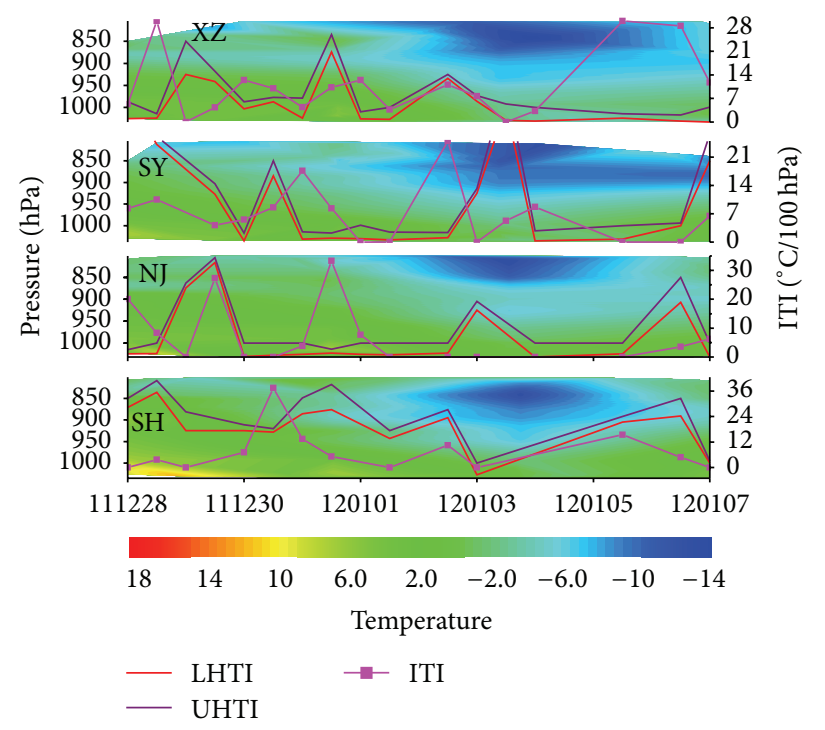

(d)

FIGURE 7: Temperature profiles of the advancing edge of a cold front (ACF) pattern during consecutive regional haze days at XuZhou (XZ), SheYang (SY), NanJing (NJ), and ShangHai (SH) ((a) 1982-12-11-1982-12-24, (b) 2003-10-24-2003-11-03, (c) 2005-01-11-2005-01-20, and (d) 2011-12-28-2012-01-07). Lower height of temperature inversions (LHTI), upper height of temperature inversions (UHTI), and intensities of the temperature inversions (ITI).

were at low altitudes. The intensity of the temperature inversion gradually increased in the early stage. In the middle to late period, the intensity gradually decreased. The intensity of the temperature inversion was strong and variable. The thickness of the temperature inversion did not noticeably change.

From 2003-10-24 to 2003-11-03 (Figure 7(b)), when consecutive haze days began, no temperature inversion appeared at the XZ and SY stations, but the surface layer of the temperature inversion was recorded at $\mathrm{NJ}$ and $\mathrm{SH}$, which experienced another layer at $1000 \mathrm{hPa}$. Following the developing of haze days, the surface layer of the temperature inversion was recorded at all 4 stations. At that time, there were multiple layers of temperature inversions. The top height of the temperature inversion had distinct diurnal variation: the top height was higher in the daytime and lower at night. Therefore, the thickness of the temperature inversion was also diurnally variable. During the entire process, the intense variation in the temperature inversion was strong.

From 2005-01-11 to 2005-01-20 (Figure 7(c)), when a persistent haze period began, the surface layer of the temperature inversion was recorded at the XZ and SY stations. There was no temperature inversion or higher layers at the $\mathrm{NJ}$ and $\mathrm{SH}$ stations. During the middle of the period, there were multiple temperature inversions from the surface to the middle level. The intensity of the temperature inversion was strong and diurnal.

From 2011-12-28 to 2012-01-07 (Figure 7(d)), the 4 stations all recorded temperature inversions when the haze period 
TABLE 5: Typical processes of the base of high-pressure $(\mathrm{BOH})$ pattern during consecutive regional haze days.

\begin{tabular}{lccc}
\hline Series & Start day & End day & Duration \\
\hline 1 & $2003-03-21$ & $2003-03-26$ & 6 \\
2 & $2003-11-11$ & $2003-11-15$ & 5 \\
3 & $2004-01-27$ & $2004-01-31$ & 5 \\
4 & $2005-09-14$ & $2005-09-20$ & 7 \\
5 & $2009-09-02$ & $2009-09-08$ & 7 \\
6 & $2010-10-06$ & $2010-10-10$ & 5 \\
\hline
\end{tabular}

began. The height of the layer diurnally varied. In longduration haze cases, there were multiple thin temperature inversion layers. The intensity of the temperature inversion was strong and variable.

During haze cases with the ACF pattern, the temperature inversion was not notable; however, as the cold front advanced, the surface layer remained until the end of the haze case. In the middle of the haze case, there were multiple temperature inversions at low and high levels. These features allowed for the 10-day haze cases. The height of the temperature inversion layer had obvious changes: it was lower in the daytime and higher at night. The intensity of the temperature inversion was strong and variable.

(3) $\mathrm{BOH}$. The distinguishing feature of the $\mathrm{BOH}$ pattern was the short-duration haze cases (Table 5).

From 2003-03-21 to 2003-03-26 (Figure 8(a)), when the haze case began, a temperature inversion up to $900 \mathrm{hPa}$ was recorded at all stations except XZ during the daytime. At night, the top of the temperature inversion reached $850 \mathrm{hPa}$ at XZ and SY, and the top height decreased to $1000 \mathrm{hPa}$ at $\mathrm{NJ}$ and $\mathrm{SH}$ when the layer thinned. On the second haze day, the temperature inversion reached the surface at the $\mathrm{XZ}$ and SY stations, but the top of the temperature inversion elevated to $800 \mathrm{hPa}$ at $\mathrm{NJ}$ and $\mathrm{SH}$. Hereafter, the temperature inversion was at the surface at XZ; it gradually reached the ground at the other stations three days later. The intensity of the temperature inversion varied during long haze cases.

From 2003-11-11 to 2003-11-15 (Figure 8(b)), on the first day of haze, multiple temperature inversions were elevated from north to south. At night, the temperature inversion occurred near the surface at the $\mathrm{XZ}$ station, and the height increased to $100 \mathrm{hPa}$ at other stations. Afterward, the surfacebased temperature inversion lasted for 5 days, except for the nighttime layer on November 13, which was elevated to $925 \mathrm{hPa}$. The intensity of the temperature inversion greatly changed. The peak of the intensity occurred on the third day of the persistent haze case, when SAP and cold air were present. Then, multiple temperature inversion layers occurred at the 4 stations.

From 2004-01-27 to 2004-01-31 (Figure 8(c)), a temperature inversion formed near the surface and lasted until the end of the haze case; however, the base of the temperature inversion at the NJ station increased to $900 \mathrm{hPa}$ at night on January 27. The intensity of the temperature inversion appeared to increase from north to south during the daytime of January 27 to the night of January 28. During the daytime of January 30, a second peak appeared at XZ and NJ. Then, the SY station experienced obvious diurnal variation while the $\mathrm{SH}$ station experienced weak diurnal variation.

From 2005-09-14 to 2005-09-20 (Figure 8(d)), when the haze case began, a temperature inversion appeared at all stations except NJ; double inversions occurred at the $\mathrm{XZ}$ and SY stations. Afterward, the base of the temperature inversion was below $950 \mathrm{hPa}$, but it increased to $850 \mathrm{hPa}$ between the daytime on September 17 and the nighttime on September 18 at the SH station. The intensity peak of the temperature inversion appeared at the $\mathrm{SH}$ station on September 15 during the day $\left(0.33^{\circ} \mathrm{C} / \mathrm{hPa}\right)$. At the other stations, the peak intensity appeared from September 16 at night to September 17 during the day, with intensity between 0.15 and $0.22^{\circ} \mathrm{C} / \mathrm{hPa}$. Specifically, the temperature inversion was thin and was mainly a single layer.

From 2009-09-02 to 2009-09-08 (Figure 8(e)), when the haze case began, a temperature inversion occurred at all stations except SY. The height increased from north to south and over time at the 4 stations. Afterward, the temperature inversion became increasingly shallow between September 3 and September 5. Between September 7 and September 8, the temperature inversion did not continue to elevate. The base of the temperature inversion was positively related to the inversion intensity: that is, when the base was higher, the intensity was strong and vice versa. The variation in the thickness was inhomogeneous and greatly different among the stations. Additionally, the layer was thin at the stations.

From 2010-10-06 to 2010-10-10 (Figure 8(f)), when the haze period began, the temperature inversion was low. The base height dropped from north to south. Afterward, the temperature inversion was at the surface at XZ. The layer ascended twice at the SY station on October 7 and October 9; at other times, the temperature inversion was at the surface. At the $\mathrm{NJ}$ station, the base of the temperature inversion increased to $950 \mathrm{hPa}$ during the daytime of October 8. At the SH station, a temperature inversion occurred during the daytime of October 9 and October 10; the inversion ascended at other times. During this process, the inversion was shallow at the 4 stations. The intensity of the temperature inversion was weak at XZ; the intensity peaked on the night of October 8. The intensity was strong in the daytime and weak at nighttime at SY; the opposite occurred at the NJ station. The intensity gradually receded during the haze days. The inversion was uniformly shallow with this type of weather pattern.

Because of the changing velocity of the system during consecutive haze days, there were larger height differences from south to north. Following haze development, the shallow temperature inversion slightly ascended while the intensity was strong.

(4) $B A H$. The representative processes of the BAH pattern during persistent regional haze are shown in Table 6. From the table, we found that the duration was short with this type of pattern.

From 1989-05-27 to 1989-06-02 (Figure 9(a)), when the haze case began, only 1 station had a temperature inversion. Although the temperature inversion developed soon 


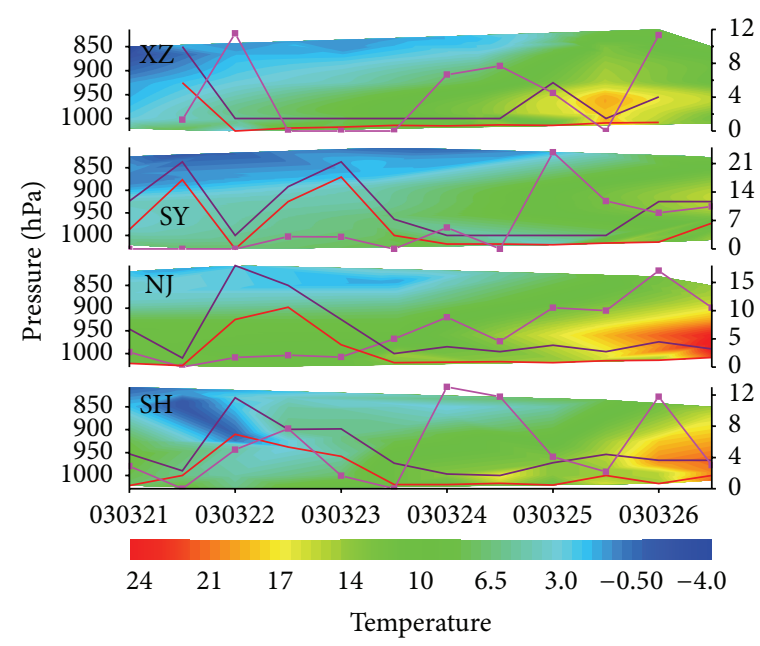

(a)

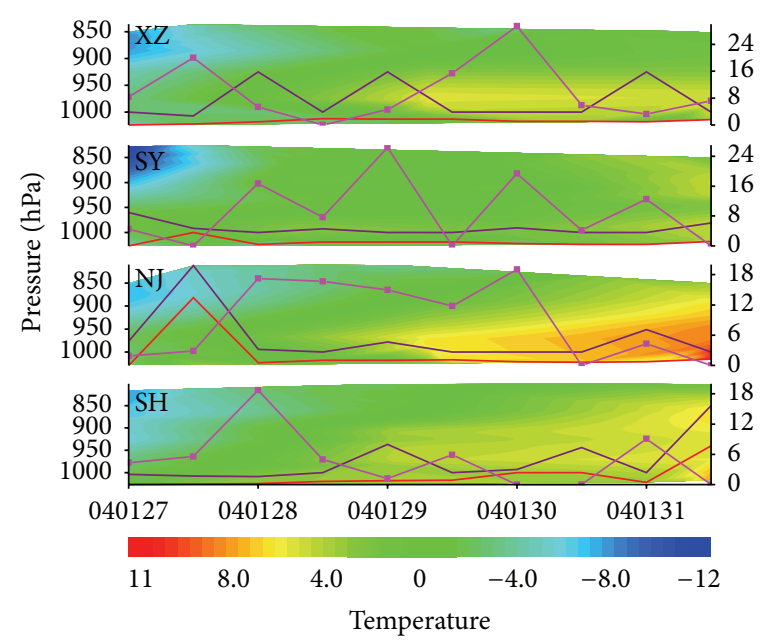

(c)

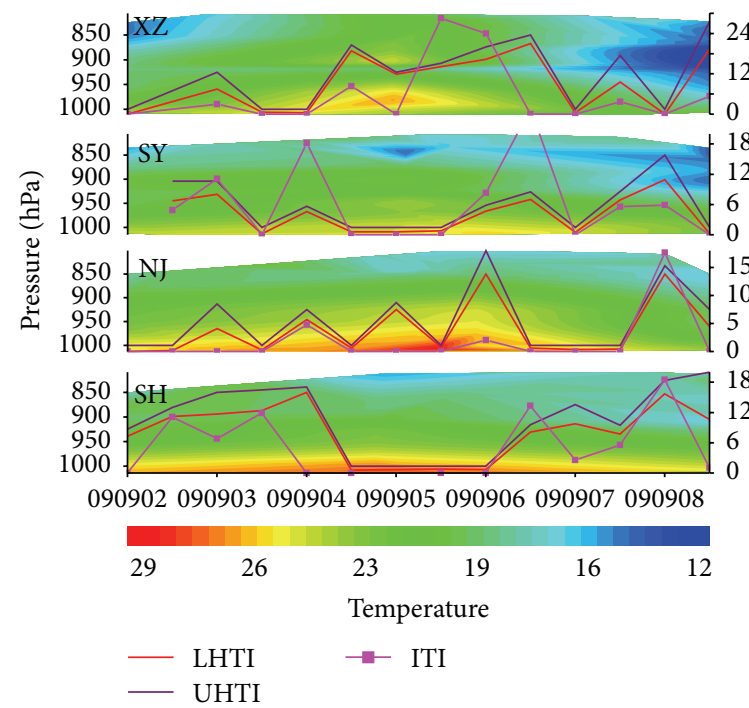

(e)

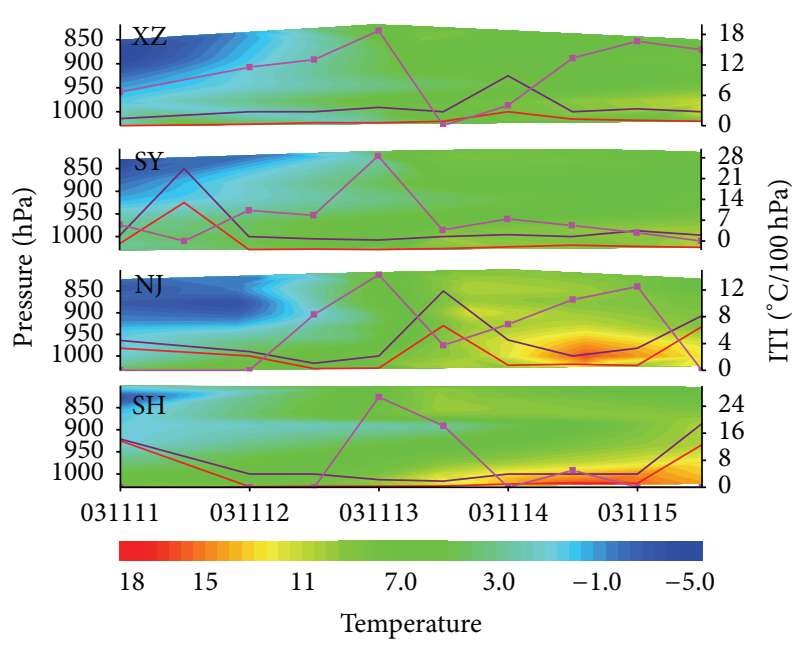

(b)

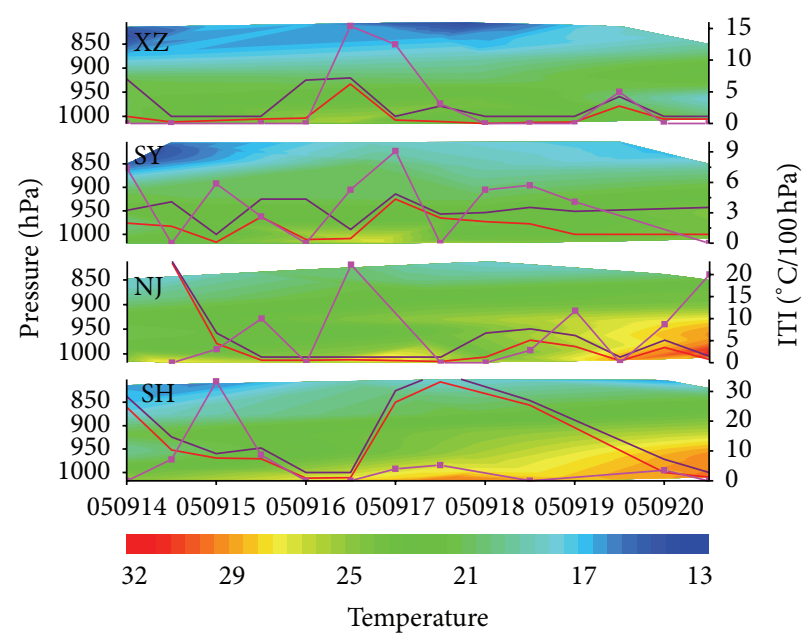

(d)

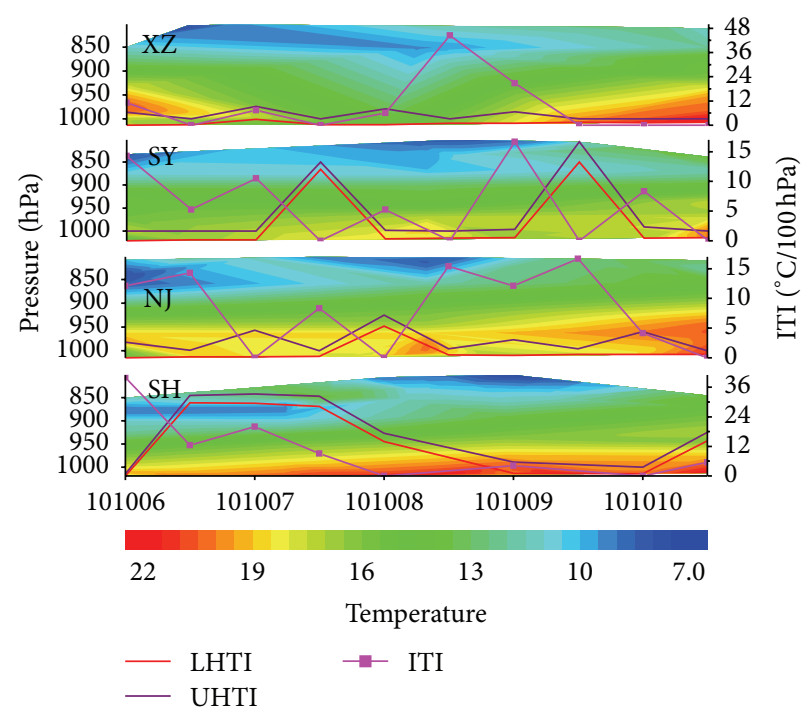

(f)

FIGURE 8: Temperature profiles of the base of high-pressure $(\mathrm{BOH})$ pattern during consecutive regional haze days at XuZhou (XZ), SheYang (SY), NanJing (NJ), and ShangHai (SH) ((a) 2003-03-21-2003-03-26, (b) 2003-11-11-2003-11-15, (c) 2004-01-27-2004-01-31, (d) 2005-09-142005-09-20, (e) 2009-09-02-2009-09-08, and (f) 2010-10-06-2010-10-10). Lower height of temperature inversions (LHTI), upper height of temperature inversion (UHTI), and intensities of the temperature inversions (ITI). 


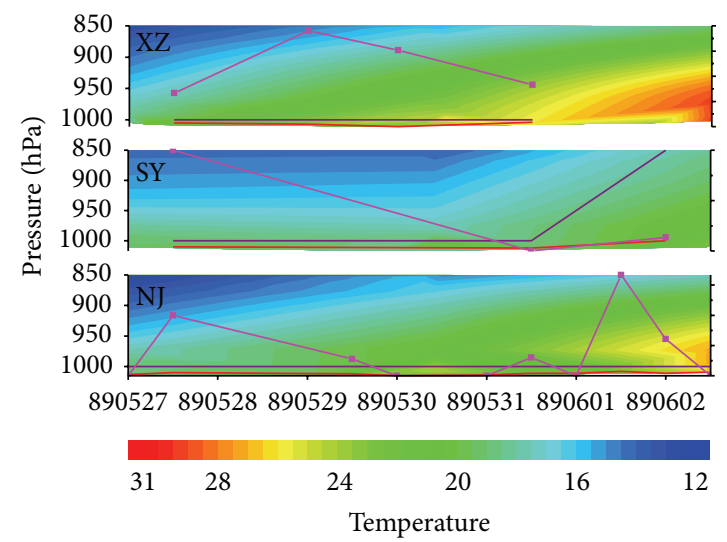

(a)

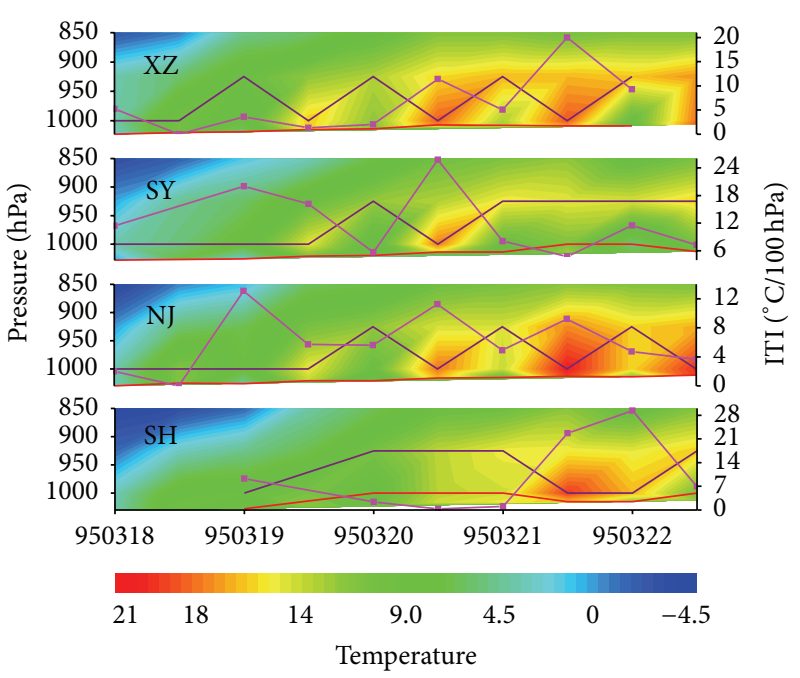

(b)

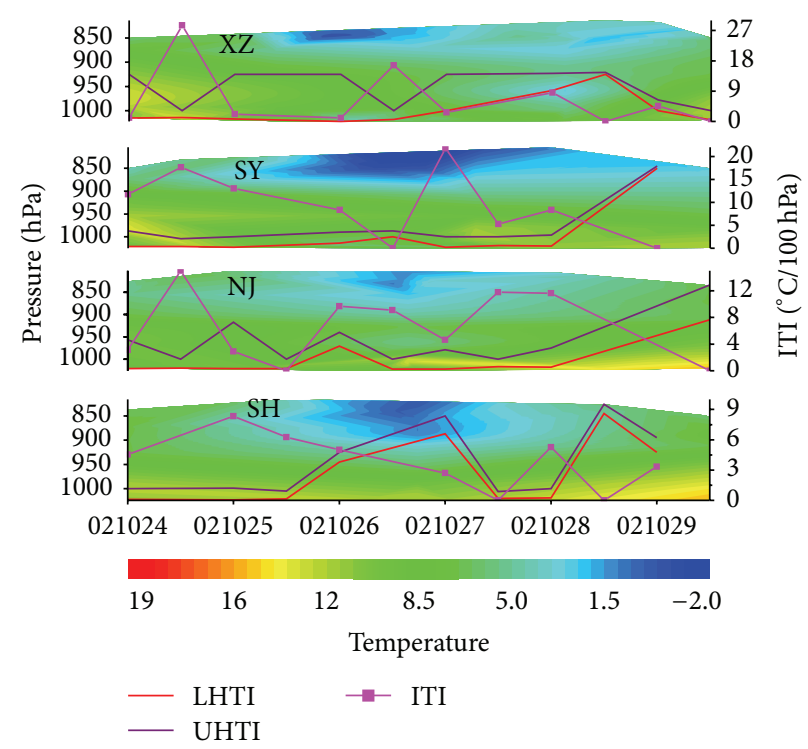

(c)

FIgURE 9: Temperature profiles of the backside of high-pressure (BAH) pattern during consecutive regional haze days at XuZhou (XZ), SheYang (SY), NanJing (NJ), and ShangHai (SH) ((a) 1989-05-27-1989-06-02, (b) 1995-03-18-1995-03-22, and (c) 2002-10-24-2002-10-29). Lower height of a temperature inversion (LHTI), upper height of a temperature inversion (UHTI), and intensity of the temperature inversion (ITI).

TABLE 6: Typical processes of the backside of high-pressure (BAH) pattern during consecutive regional haze days.

\begin{tabular}{lccc}
\hline Series & Start day & End day & Duration \\
\hline 1 & $1989-05-27$ & $1989-06-02$ & 7 \\
2 & $1995-03-18$ & $1995-03-22$ & 5 \\
3 & $2002-10-24$ & $2002-10-29$ & 6 \\
\hline
\end{tabular}

afterward, the duration was short. The temperature inversion was above the surface, weak, and thin.

From 1995-03-18 to 1995-03-22 (Figure 9(b)), the temperature inversion was just above the surface. The thickness had a notable diurnal variation: thick in the daytime and thin at night. The intensity of the temperature varied among the 4 stations: it was stronger at night than during the day (opposite effect with the thickness). The intensity gradually increased at XZ following the haze development, but it gradually decreased at NJ. The peak of the intensity occurred overnight on March 20 at SY and during the daytime on March 22 at $\mathrm{SH}$.

From 2002-10-24 to 2002-10-29 (Figure 9(c)), when the haze days began, a temperature inversion occurred near the surface, and the base of the temperature inversion increased to $950 \mathrm{hPa}$ on the night of October 28 at XZ. The base of the temperature inversion increased on October 25 and the night of October 28 at SY; the inversion reached $850 \mathrm{hPa}$ during the day on October 29. The base of the temperature 
inversion reached $1000 \mathrm{hPa}$ during the daytime on October 26 and nighttime of October 29 at NJ. The temperature inversion was generally higher after October 26 . The thickness of the temperature inversion was variable: it was thick initially and thinned later. The base of the temperature varied in opposition to the intensity.

The surface layer of the temperature inversion was a major feature of the BAH pattern. Initially, the temperature inversion was thick; with the development of haze, the layer thinned and weakened.

\subsubsection{Boundary-Layer Structures during Regional Heavy Haze}

(1) EQP. In total, 12 days had regional heavy haze during the EQP pattern, 8 of which occurred in autumn or winter.

The boundary layer had 4 distinct structures. First, there was an inversion layer on the surface or an isothermal layer at all 4 stations (Figures 10(c), 10(d), 10(e), 10(f), 10(g), 10(h), and $10(\mathrm{j})$ ); Figure 10(c) displays the single structure. The inversion layer or the isothermal layer structure occurred at $800 \mathrm{hPa}$ in Figures 10(d), 10(e), 10(f), 10(g), and 10(h); at this height, the temperature inversion layer was thin and weak. A stronger inversion layer occurred between $850 \mathrm{hPa}$ and $925 \mathrm{hPa}$ in Figure 10(j). Second, 3 stations had inversion layers that occurred near the surface (Figures 10(b) and 10(k)), and only 1 station had a temperature inversion or isothermal structure between $925 \mathrm{hPa}$ and $975 \mathrm{hPa}$. Third, 2 stations had inversion layers near the ground. Although no ground inversion layer occurred at the SY station, a thick temperature inversion layer occurred at $1000 \mathrm{hPa}$. Fourth, no apparent inversion layer existed, but a weak structure occurred at $975 \mathrm{hPa}$ at the $\mathrm{SH}$ station in Figure 10(i).

This weather pattern was mainly characterized by a single or double inversion layer or isothermal layer near the ground. No inversion layer was present, only Figure 10(i) process. Generally, the atmospheric stability was slightly weak in summer, so stable stratification had difficulty forming. Therefore, we believe that heavy pollution was mainly caused by the sudden emissions release locally or overseas; because the air purification was insufficient, heavy haze formed.

(2) ACF. All instances of this weather pattern were associated with temperature inversions, but differences in height occurred. The inversion layer or the isothermal layer may have been at the surface (Figures 11(a), 11(h), and 11(i)), while other cases had multiple inversion layers (Figures 11(h) and $11(\mathrm{i})$ ) between $800 \mathrm{hPa}$ and $1000 \mathrm{hPa}$. Some cases had a surface temperature inversion at 3 stations (Figures 11(b) and 11(c)). Although one of the 4 stations had no temperature inversion at the surface (it occurred between $975 \mathrm{hPa}$ and $1000 \mathrm{hPa}$ ). Some cases had a surface-layer temperature inversion at 2 stations (Figures 11(d), 11(e), and 11(f)); however, at the other 2 stations, an inversion layer occurred between $900 \mathrm{hPa}$ and $1000 \mathrm{hPa}$. Figure $11(\mathrm{j})$ shows that only 1 station had a surface-based temperature inversion, while the other 3 stations had inversions between $850 \mathrm{hPa}$ and $1000 \mathrm{hPa}$. Figure $11(\mathrm{~g})$ shows that a surface-based inversion did not occur at any of the stations, but the temperature inversion occurred between $925 \mathrm{hPa}$ and $1000 \mathrm{hPa}$, except at the SY station.

The ACF pattern shows temperature inversions at different heights; the main feature was a single-layer temperature inversion and a high-altitude isothermal layer.

(3) $\mathrm{BOH}$. A temperature inversion occurred near the surface at all 4 stations (Figures 12(c) and 12(d)), and there were multiple layers of temperature inversions between $800 \mathrm{hPa}$ and $975 \mathrm{hPa}$ (Figure 12(d)). Figure 12(a) shows a temperature inversion at the surface at 3 stations; the remaining station had an inversion at $1000 \mathrm{hPa}$. In some cases, 2 stations had temperature inversions at the surface, while the inversions occurred between $950 \mathrm{hPa}$ and $1000 \mathrm{hPa}$ at the other 2 stations. Figure 12(b) shows that only 1 station had a surface temperature inversion, while the other 3 stations had temperature inversions between $950 \mathrm{hPa}$ and $1000 \mathrm{hPa}$.

In this weather pattern, the height of the temperature inversion layer greatly varied. However, all of the inversion layers formed below $950 \mathrm{hPa}$.

(4) BAH. Most of the cases were similar (Figures 13(a), 13(b), 13(d), 13(e), 13(f), 13(g), and 13(i)), with inversion layers or isothermal layers at the surface. Some cases had multiple temperature inversion layer structures (Figures 13(a), 13(b), 13(d), 13(e), 13(f), and 13(i)). Figure 13(h) shows temperature inversions and isothermal layers at the surface at all stations except XZ. Figure 13(c) shows that the temperature inversion layer was at $1000 \mathrm{hPa}$ at the $\mathrm{XZ}$ and SY stations only. Figure 13(j) shows that the temperature inversion occurred at the surface at the $\mathrm{NJ}$ and $\mathrm{SH}$ stations and at $980 \mathrm{hPa}$ at the $\mathrm{XZ}$ and SY stations.

This type of heavy haze case is associated with an inversion layer or isothermal layer on the ground; most cases had multiple inversion structures.

(5) INT. All of the cases of INT were similar at the 4 stations (Figures 14(a), 14(d), and 14(e)). The layer of the temperature inversion was above $1000 \mathrm{hPa}$, and the base of the inversion layer was also coincident. Figures 14(b) and 14(f) show that multiple temperature inversions occurred at the surface. Figure 14(c) reveals that the base of the temperature inversion was at $1000 \mathrm{hPa}$ at the $\mathrm{NJ}$ station and was near the surface at the other stations.

The temperature inversion was thick and elevated in these cases.

(6) Other Weather Patterns. Figures 15(a) and 15(c) show an inversion and isothermal layer at the surface and multilayer temperature inversions. Figure 15(b) reveals the isothermal layer at the surface. Figure 15(d) shows the isothermal layer at the surface at the SY and XZ stations; the structure of the temperature inversion was not obvious. Figure 15(e) shows the isothermal layer at the surface and the thin inversion layer at $980 \mathrm{hPa}$. The cases in Figures 15(b), 15(d), and 15(e) occurred in late spring or early summer (May to June). The structure of the temperature inversion was not significant, and the temperature inversion was thin. Therefore, the cause 


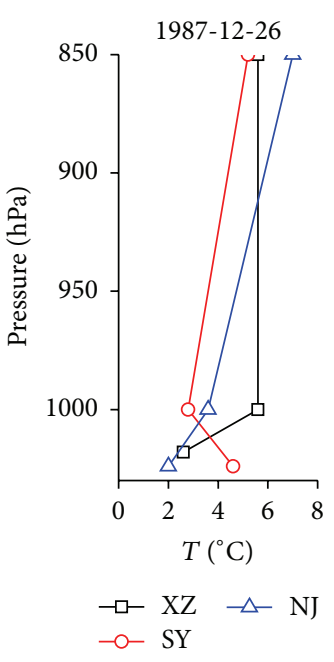

(a)

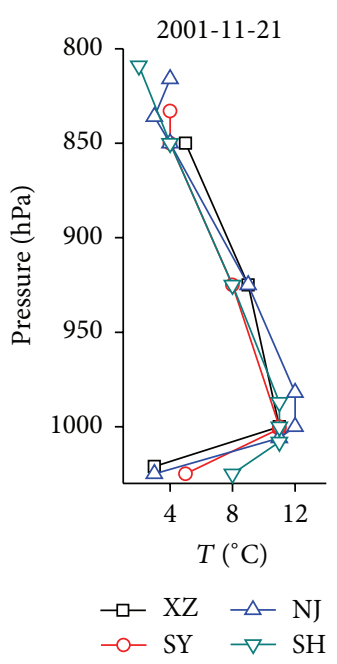

(e)

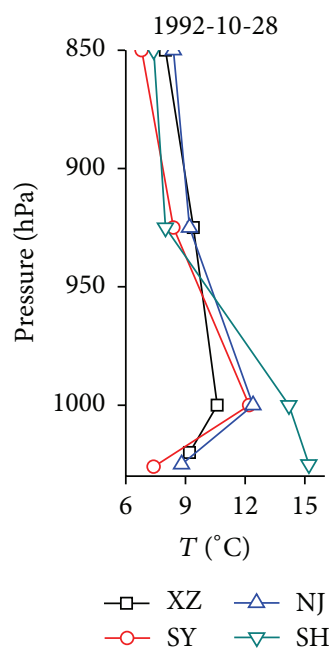

(b)

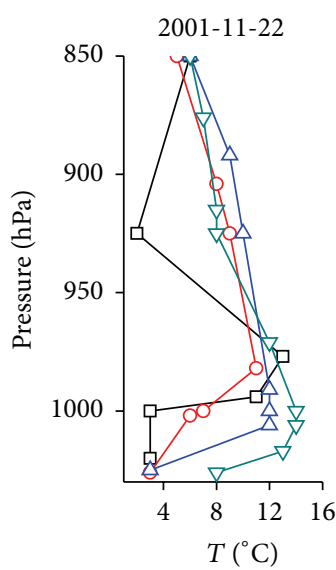

$$
\begin{array}{ll}
\rightarrow-\mathrm{XZ} & -\backsim \mathrm{NJ} \\
\multimap-\mathrm{SY} & -\nabla-\mathrm{SH}
\end{array}
$$

(f)

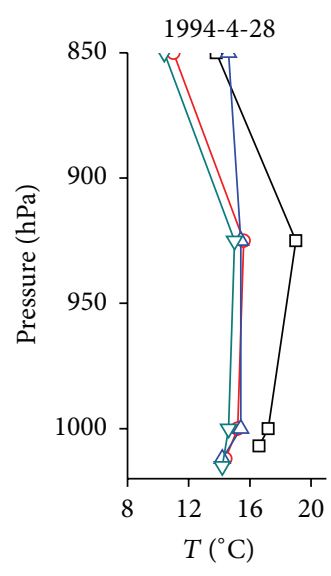

$$
\rightarrow-\mathrm{XZ} \quad \triangle-\mathrm{NJ}
$$$$
-\mathrm{OSY} \quad-\nabla-\mathrm{SH}
$$

(c)

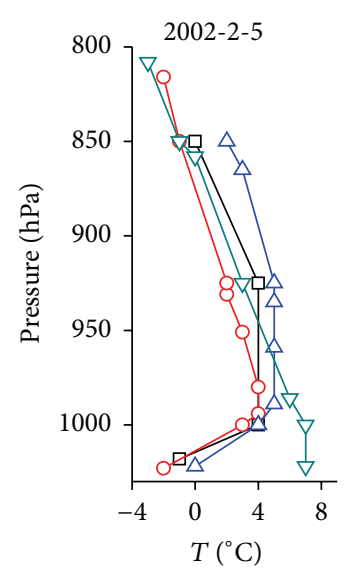

$\begin{array}{ll}\rightarrow \mathrm{XZ} & -\triangle \mathrm{NJ} \\ -\mathrm{O} \mathrm{SY} & \rightarrow-\mathrm{SH}\end{array}$

(g)

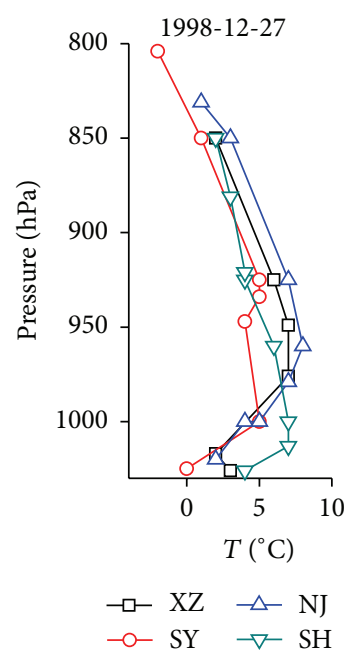

(d)

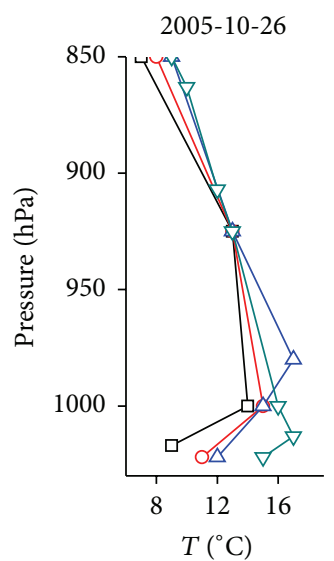

$$
\begin{array}{ll}
\rightarrow-\mathrm{XZ} & -\checkmark \mathrm{NJ} \\
\multimap-\mathrm{SY} & -\nabla-\mathrm{SH}
\end{array}
$$

(h)

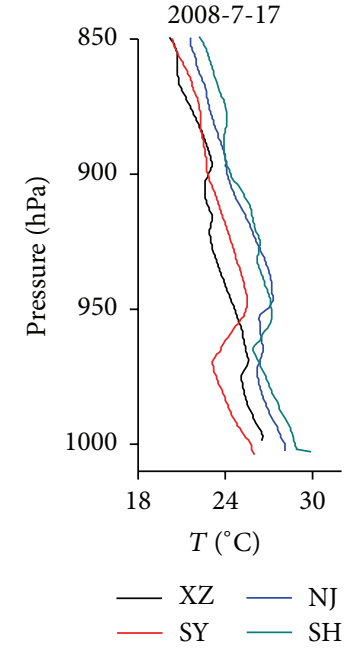

(i)
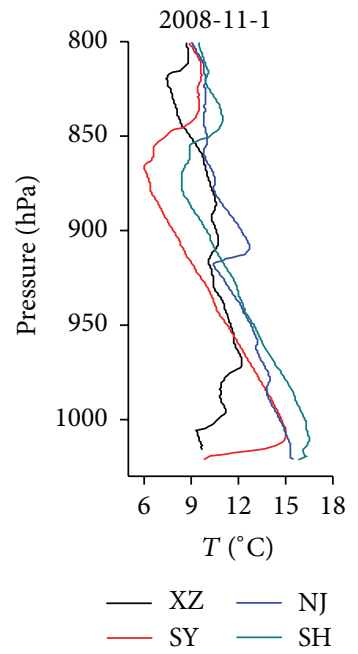

(j)

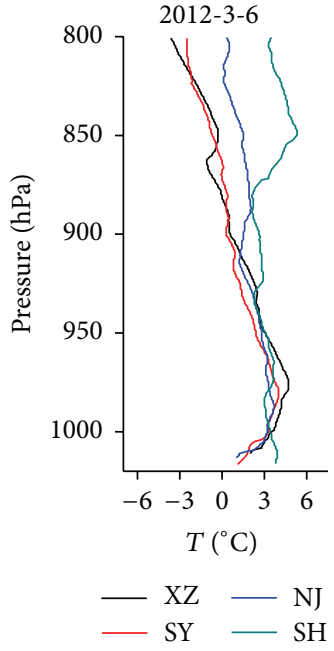

(k)

FIGURE 10: Temperature profiles of the EQP pattern during regional heavy haze days at XuZhou (XZ), SheYang (SY), NanJing (NJ), and ShangHai (SH). The $x$-axis is the temperature, the $y$-axis is the pressure, and the curved lines are the temperature profiles at the sounding stations ((a) 1987-12-26, (b) 1992-10-28, (c) 1994-04-28, (d) 1998-12-27, (e) 2001-11-21, (f) 2001-11-22, (g) 2002-02-05, (h) 2005-12-26, (i) 200807-17, (j) 2008-11-01, and (k) 2012-03-06). 

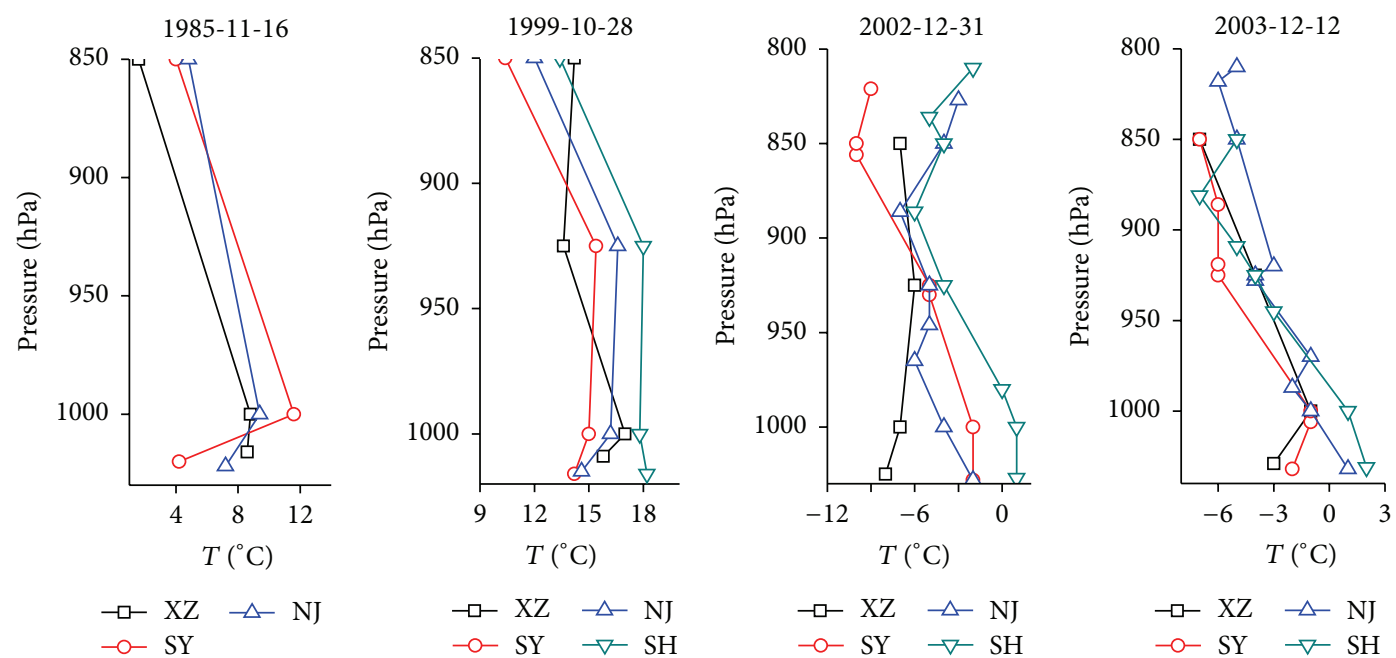

$$
\neg-\mathrm{XZ} \quad \triangle \mathrm{NJ}
$$

(a)

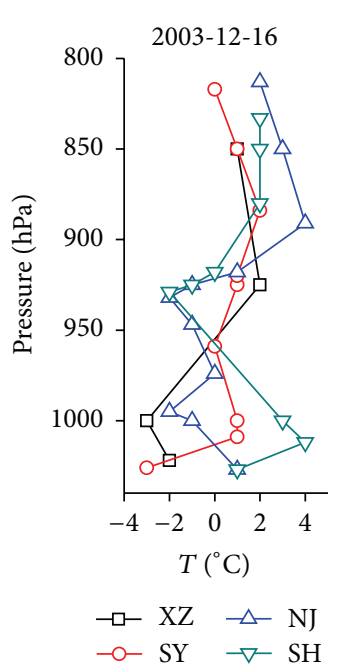

(b)

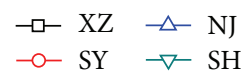

$$
\neg-\mathrm{XZ} \quad \triangle-\mathrm{NJ}
$$

(c)

(d)
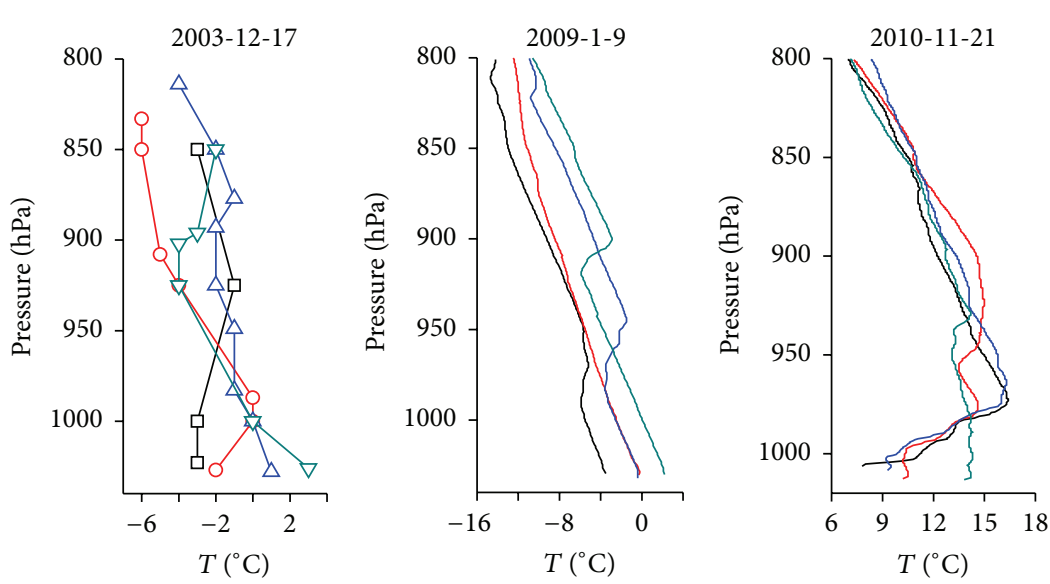

$-\mathrm{O}-\mathrm{SY} \quad-\mathrm{-}-\mathrm{SH}$
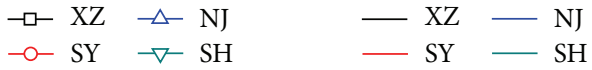

(g)

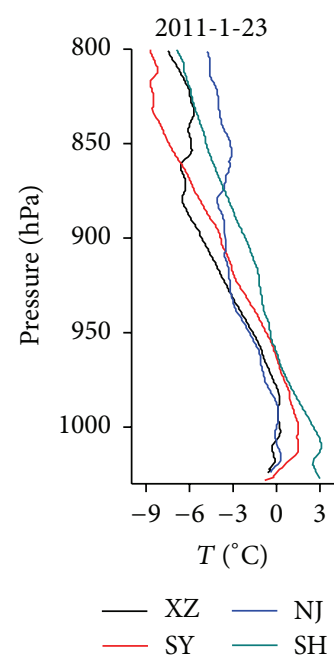

(i)

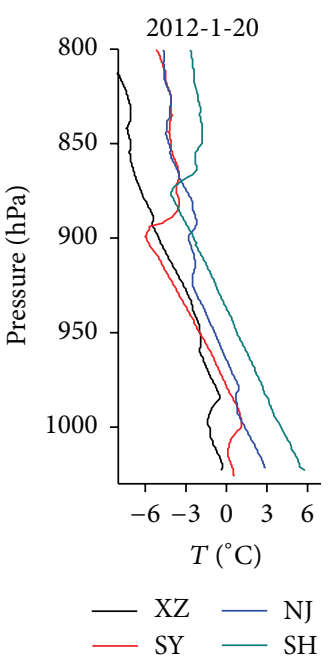

(j)

FIGURE 11: Temperature profiles of the ACF pattern during regional heavy haze days at XuZhou (XZ), SheYang (SY), NanJing (NJ), and ShangHai (SH). The $x$-axis is temperature, the $y$-axis is pressure, and the curved lines are the temperature profiles at the sounding stations ((a) 1985-11-16, (b) 1999-10-28, (c) 2002-12-31, (d) 2003-12-12, (e) 2003-12-16, (f) 2003-12-27, (g) 2009-01-09, (h) 2010-11-21, (i) 2011-01-23, and (j) 2012-01-20). 


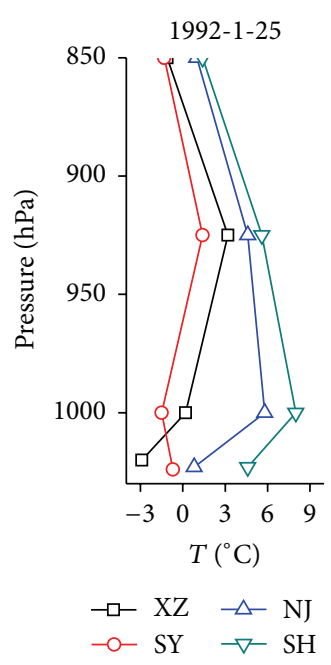

(a)

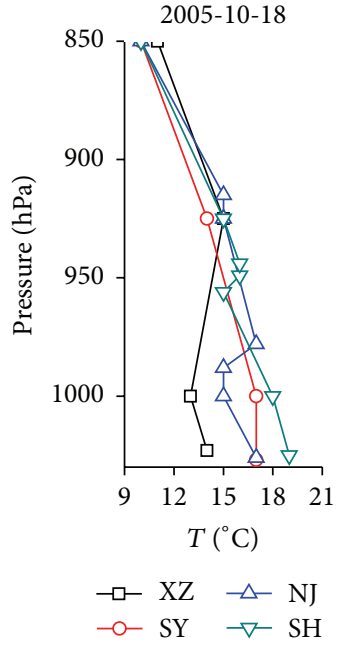

(b)

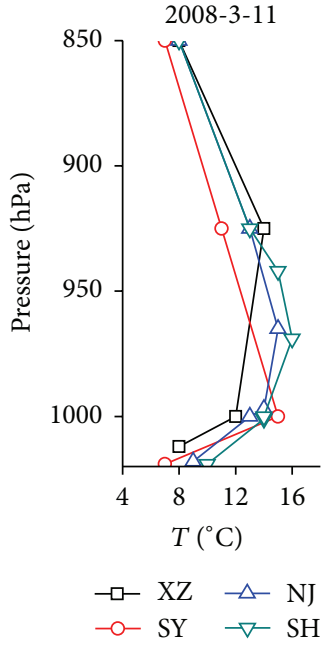

(c)

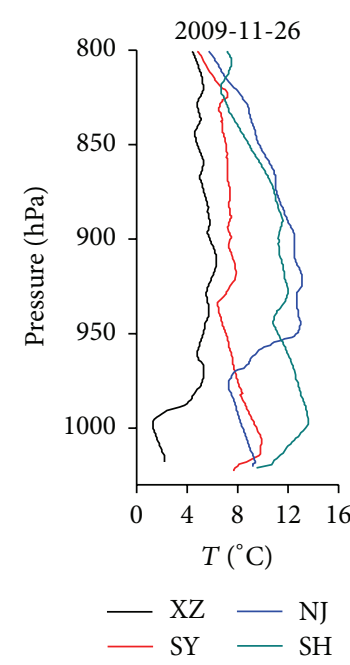

(d)

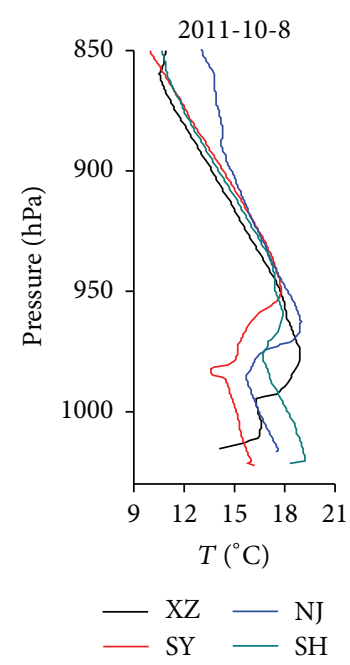

(e)

FIGURE 12: Temperature profiles of the BOH pattern during regional heavy haze days at XuZhou (XZ), SheYang (SY), NanJing (NJ), and ShangHai (SH). The $x$-axis is temperature, the $y$-axis is pressure, and the curved lines are the temperature profiles at the sounding stations ((a) 1992-01-25, (b) 2005-10-18, (c) 2008-03-11, (d) 2009-11-26, and (e) 2011-10-08).

of the heavy haze may be regional summer harvesting and seeding [64].

\section{Conclusions}

Existing research focuses on spatiotemporal haze distributions or the synoptic situation and boundary-layer characteristics of specific case studies. However, the weather and boundary-layer climate over many years are not well-known, particularly in terms of persistent haze and heavy haze. In this study, we classify haze and associated weather systems in southern Jiangsu from 1980 to 2012; the boundary-layer characteristics of regional haze events and regional heavy haze were also studied. The main conclusions are as follows:

(1) The surface conditions were dominated by equalized pressure (EQP), the advancing edge of a cold front
(ACF), the base of high pressure $(\mathrm{BOH})$, the backside of high pressure (BAH), the inverted trough of low pressure (INT), and saddle pressure (SAP).

(2) Based on the surface conditions, regional haze events could be divided into 4 types, each of which has a different boundary-layer structure. Type I is EQP, in which the boundary layer is that the bottom height of the temperature inversion layer is lower, the thickness is thinner, and the intensity is diverse with the different cases. Type II is the ACF, in which a cold front gradually advances and creates a nearsurface temperature inversion in most processes. The intensity of the temperature inversion was strong and varied. Type III is the $\mathrm{BOH}$, in which a shallow but intense temperature inversion is elevated as haze event progresses. Type IV is the BAH, at the start of 

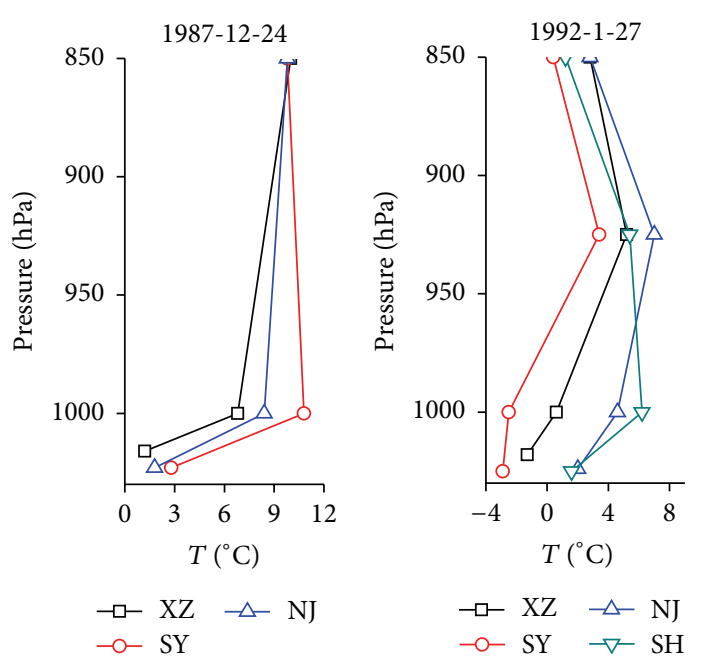

(b)

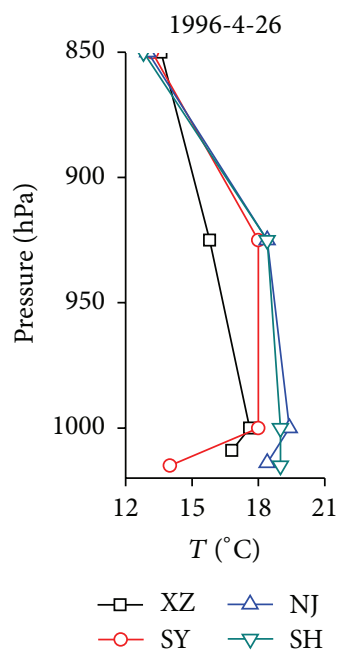

(e)

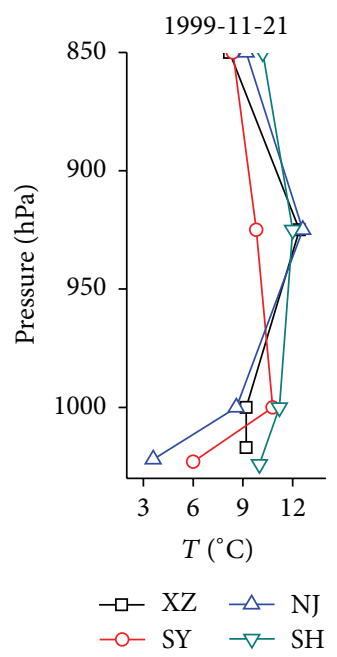

(f)

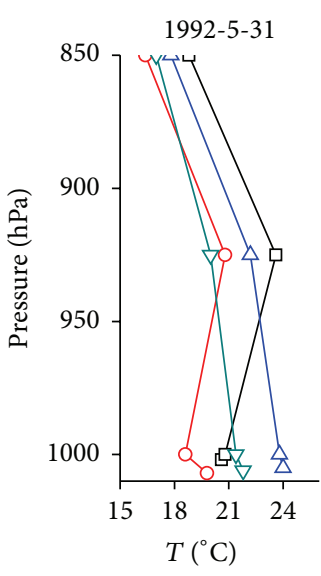

$\neg-\mathrm{XZ} \quad-\mathrm{NJ}$

$-\mathrm{O} S \mathrm{SY} \quad-\nabla-\mathrm{SH}$

(c)

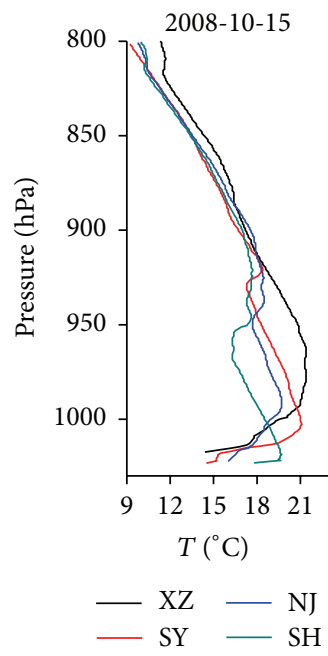

(i)

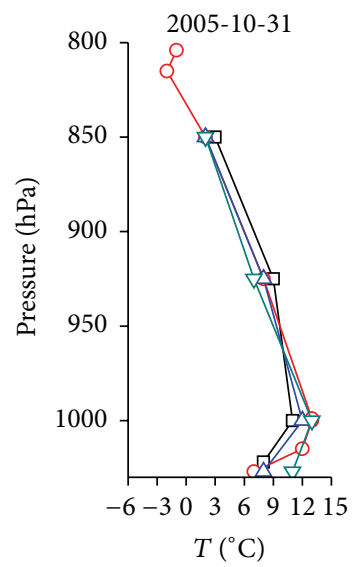

$$
\begin{array}{ll}
\rightarrow-\mathrm{XZ} & \rightarrow \mathrm{NJ} \\
\rightarrow \mathrm{SY} & \rightarrow-\mathrm{SH}
\end{array}
$$

(g)
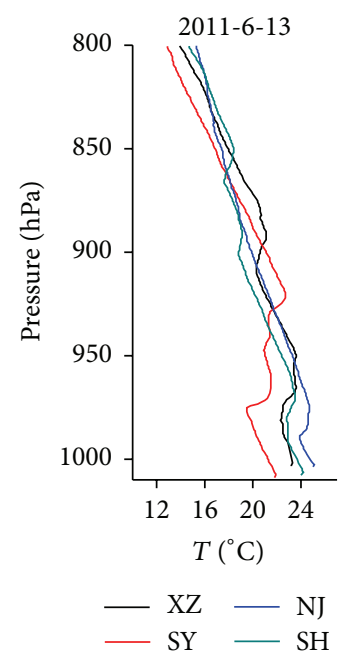

(j)

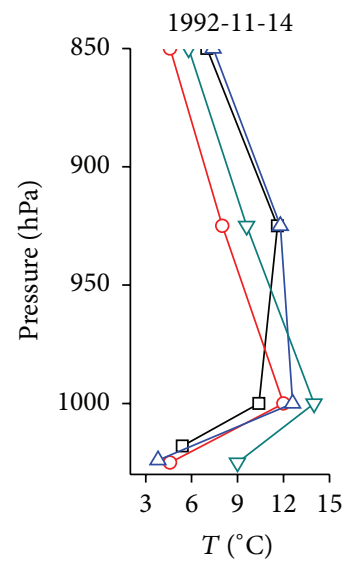

$$
\begin{array}{ll}
\rightarrow-\mathrm{XZ} & -\backsim \mathrm{NJ} \\
-\mathrm{S} & \rightarrow-\mathrm{SH}
\end{array}
$$

(d)

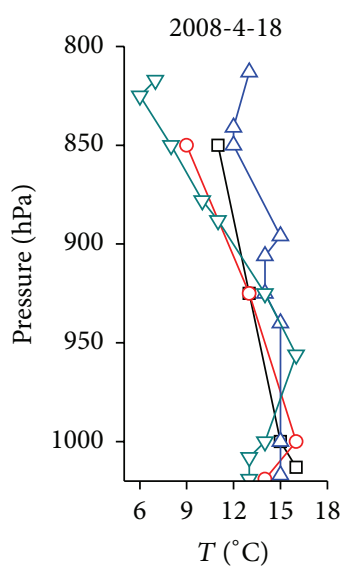$$
\neg-\mathrm{XZ} \quad-\triangle \mathrm{NJ}
$$

(h)

FIGURE 13: Temperature profiles of the BAH pattern during regional heavy haze days at XuZhou (XZ), SheYang (SY), NanJing (NJ), and ShangHai (SH). The $x$-axis is temperature, the $y$-axis is pressure, and the curved lines are the temperature profiles at the sounding stations ((a) 1987-12-24, (b) 1992-01-27, (c) 1992-05-31, (d) 1992-11-11, (e) 1996-04-26, (f) 1999-11-21, (g) 2005-10-31, (h) 2008-04-18, (i) 2008-10-15, and (j) 2011-06-13). 


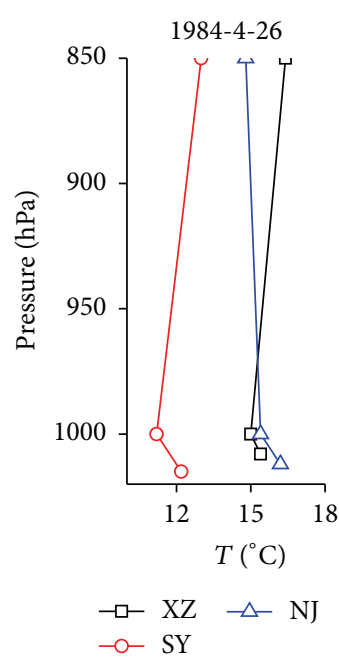

(a)

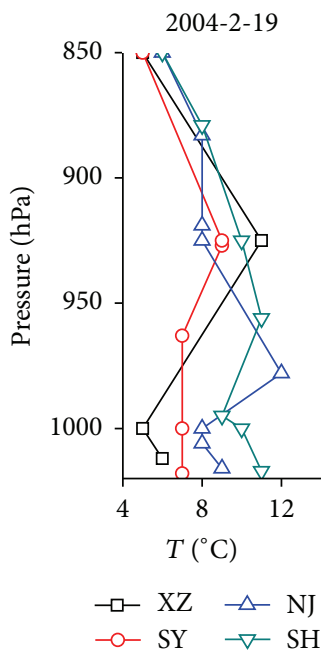

(d)

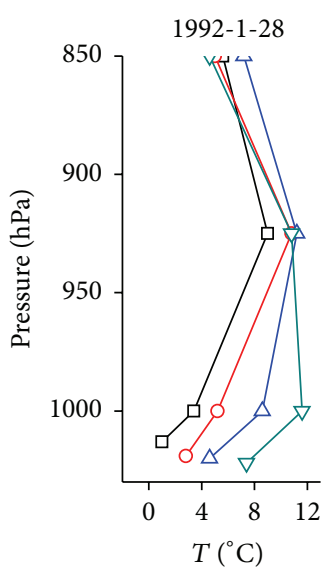

$\neg-\mathrm{XZ} \quad \triangle \mathrm{NJ}$

$\rightarrow-S Y \quad \rightarrow-S H$

(b)

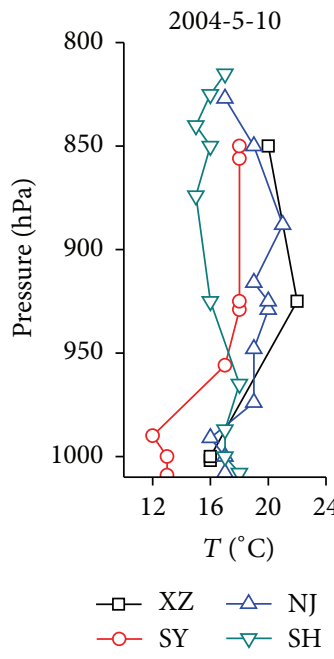

(e)

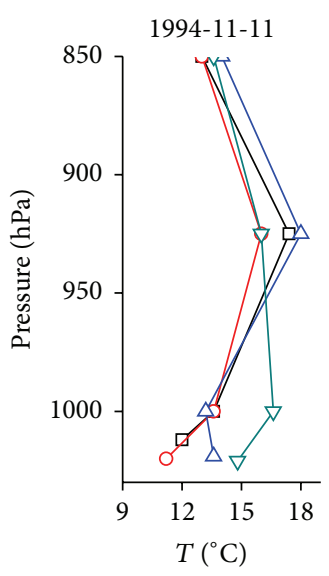

$\neg-\mathrm{XZ} \quad \triangle \mathrm{NJ}$

$-\circ$ SY $\rightarrow-S H$

(c)

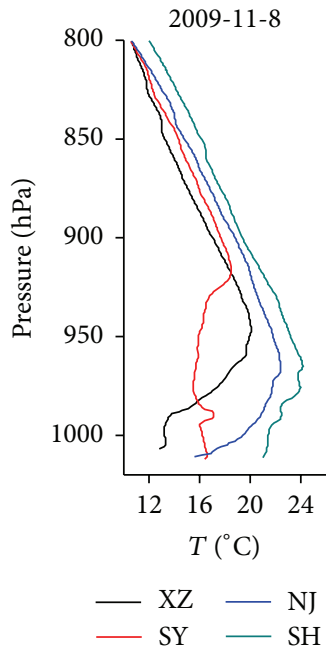

(f)

Figure 14: Temperature profiles of the INT pattern during regional heavy haze days at XuZhou (XZ), SheYang (SY), NanJing (NJ), and ShangHai (SH). The $x$-axis is temperature, the $y$-axis is pressure, and the curved lines are the temperature profiles at the sounding stations ((a) 1984-04-26, (b) 1992-01-28, (c) 1994-11-11, (d) 2004-02-19, (e) 2004-05-10, and (f) 2009-11-08).

the haze-day case, in which the temperature inversion is vertically extensive. However, as the haze event progresses, the layer becomes shallow and weak.

(3) During heavy haze, the surface mainly experiences EQP, ACF, BOH, BAH, and INT. The boundarylayer structure of EQP is characterized by a single or double inversion or isothermal layer near the surface. The ACF is associated with a unique inversion layer height, which is often a single layer. An isothermal layer is common at high altitudes. The $\mathrm{BOH}$ reveals a low-level temperature inversion. The BAH pattern displays a multilayer temperature inversion or isothermal layer at the surface. The INT has an extensive boundary layer with an elevated temperature inversion.

\section{Conflict of Interests}

The authors declare that there is no conflict of interests regarding the publication of this paper.

\section{Acknowledgments}

Funding for this work was jointly provided by the Natural Science Foundation of Jiangsu Province (Grant no. BK20130111), the Key Projects of Jiangsu Meteorological Bureau (Grant no. KZ201405), the Social Development Projects of Wuxi (Science and Technology Development) (Grant no. CSE11N1301), and the National Key Technology Support Program (Grant no. 2014BAC22B04). 


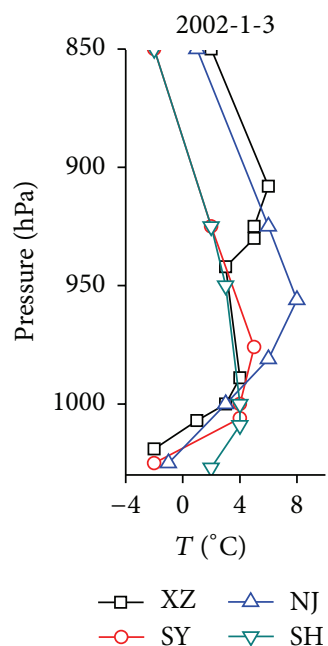

(a)

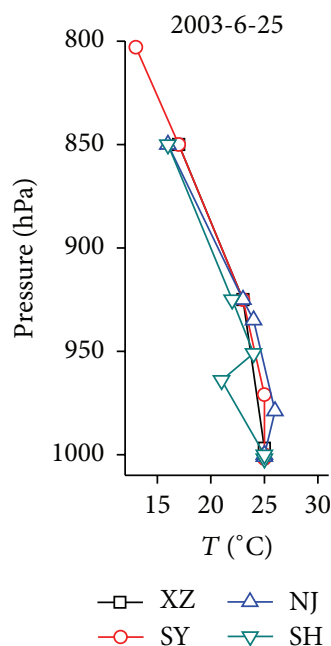

(b)

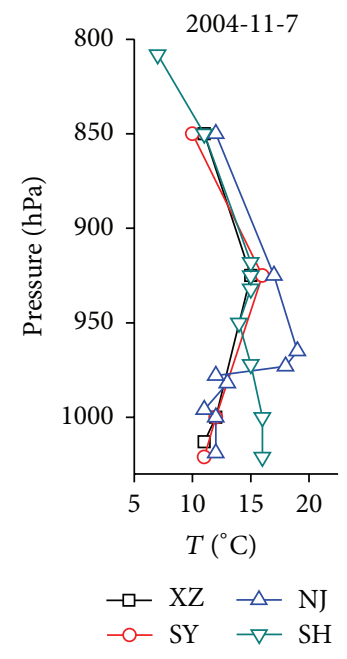

(c)

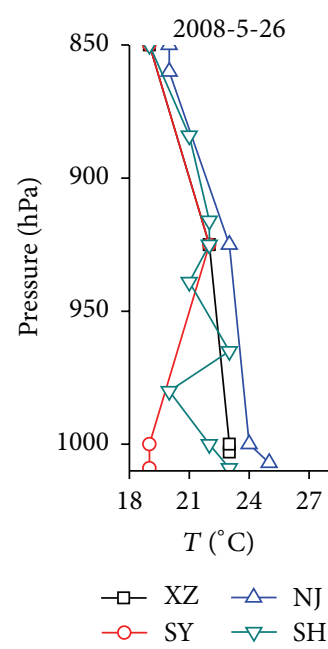

(d)

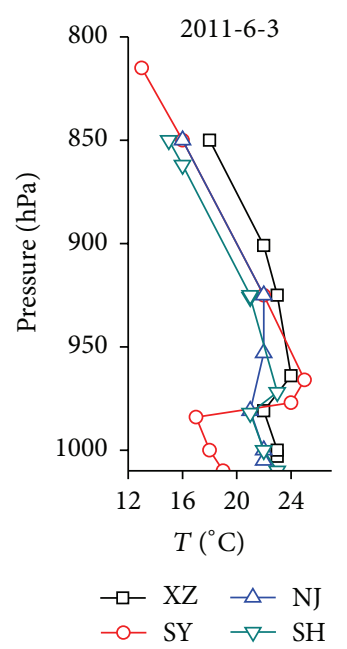

(e)

Figure 15: Temperature profiles of other weather types during regional heavy haze days at XuZhou (XZ), SheYang (SY), NanJing (NJ), and ShangHai (SH). The $x$-axis is temperature, the $y$-axis is pressure, and the curved lines are the temperature profiles at the sounding stations ((a) 2002-01-03, (b) 2003-06-25, (c) 2004-11-07, (d) 2008-05-26, and (e) 2011-06-03).

\section{References}

[1] R. G. Elridge, “Climatic visibilities of the United States," Journal of Applied Meteorology, vol. 5, pp. 227-282, 1966.

[2] M. E. Miller, N. L. Canfield, T. A. Ritter, and C. R. Weaver, "Visibility changes in Ohio, Kentucky, and Tennessee from 1962 to 1969," Monthly Weather Review, vol. 100, no. 1, pp. 67-71, 1972.

[3] R. E. Munn, "Secular increases in summer haziness in the Atlantic provinces," Atmosphere, vol. 11, pp. 156-161, 1973.

[4] R. E. Weiss, A. P. Waggoner, R. J. Charlson et al., "Sulfateaerosol: its geographical extent in the midwestern and southern United States," Science, vol. 197, pp. 997-998, 1977.

[5] R. B. Husar, D. E. Poll, J. M. Holloway et al., "Trends of eastern U.S. haziness since 1948," in Proceedings of the 4th Symposium on Turbulence, Diffusion and Air Pollution, pp. 249-256, American Meteorological Society, Boston, Mass, USA, 1979.

[6] R. B. Husar, J. M. Holloway, D. E. Patterson, and W. E. Wilson, "Spatial and temporal pattern of eastern U.S. haziness: a summary," Atmospheric Environment, vol. 15, no. 10-11, pp. 19191928, 1981.

[7] M. A. Ferman, G. T. Wolff, and N. A. Kelly, "The nature and sources of haze in the Shenandoah Valley/Blue Ridge Mountains area," Journal of the Air Pollution Control Association, vol. 31, no. 10, pp. 1074-1082, 1981.

[8] G. T. Wolff, N. A. Kelly, and M. A. Freman, "Source regions of summertime ozone and haze episodes in the eastern United States," Water, Air, and Soil Pollution, vol. 18, no. 1-3, pp. 65-81, 1982.

[9] J. Trijonis, "Existing and natural background levels of visibility and fine particles in the rural East," Atmospheric Environment, vol. 16, no. 10, pp. 2431-2445, 1982.

[10] C. S. Sloane, "Visibility trends. I. Methods of analysis," Atmospheric Environment, vol. 16, no. 1, pp. 41-51, 1982.

[11] C. S. Sloane, "Visibility trends-II. Mideastern United States 1948-1978," Atmospheric Environment, vol. 16, no. 10, pp. 23092321, 1982. 
[12] C. S. Sloane, "Summertine visibility declines: Meteorological influences," Atmospheric Environment, vol. 17, no. 4, pp. $763-$ 774, 1983.

[13] C. S. Sloane, "Meteorologically adjusted air quality trends: visibility," Atmospheric Environment Part A: General Topics, vol. 18, no. 6, pp. 1217-1229, 1984.

[14] R. B. Husar and W. E. Wilson, "Haze and sulfur emission trends in the eastern United States," Environmental Science \& Technology, vol. 27, no. 1, pp. 12-16, 1993.

[15] M. Radojevic and H. Hassan, "Air quality in Brunei Darussalam during the 1998 haze episode," Atmospheric Environment, vol. 33, no. 22, pp. 3651-3658, 1999.

[16] L. D. Zhang, W. J. Ye, D. H. Chen et al., "Discuss the haze weather impaction on the visibility of Xiamen city," Journal of Meteorological Research and Application (Chinese Edition), vol. 28, no. 11, pp. 149-150, 2007.

[17] X. L. Chen, Y. R. Feng, S. J. Fan et al., "Effect of the off-shore background flow and sea-land breezes on haze weather over the pearl river delta region," Chinese Journal of Atmospheric Sciences (Chinese Edition), vol. 32, no. 3, pp. 530-542, 2008.

[18] Y. F. Liao, X. Y. Wu, Z. X. Pan et al., "Climatic characteristics of haze in hunan province during 1961-2006," Advances in Climate Change Research (Chinese Edition), vol. 3, no. 5, pp. 260-265, 2007.

[19] Z. J. Jiang, F. X. Long, Y. Y. Li et al., "Haze variation characteristics of Guilin city in recent 10 years," Journal of Meteorological Research and Application (Chinese Edition), vol. 30, no. 1, pp. 6879, 2009.

[20] G. Gao, "The climatic characteristics and change of haze days over China during 1961-2005," Acta Geographica Sinica, vol. 63, no. 7, pp. 761-768, 2008.

[21] Y. D. Hu and Z. J. Zhou, "Climatic characteristics of haze in China," Meteorological Monthly (Chinese Edition), vol. 35, no. 7, pp. 73-78, 2009.

[22] D. Wu, X. J. Wu, F. Li et al., "Temporal and spatial variation of haze during 1951-2005 in Chinese mainland," Acta Meteorologica Sinica (Chinese Edition), vol. 68, no. 5, pp. 680-688, 2010.

[23] L. C. Song, R. Gao, Y. Li, and G. F. Wang, "Analysis of China's haze days in winter half year and climatic background during 1961-2012," Advances in Climate Change Research, vol. 9, no. 5, pp. 313-318, 2013.

[24] Z. Cheng, C. Chen, C. Huang, H. Huang, L. Li, and H. Wang, "Trans-boundary primary air pollution between cities in the Yangtze River Delta," Acta Scientiae Circumstantiae (Chinese Edition), vol. 31, no. 4, pp. 686-694, 2011.

[25] J. Shi, L. L. Cui, Q. S. He, and L. Sun, "The changes and causes of fog and haze days in eastern China," Acta Geographica Sinica, vol. 65, no. 5, pp. 533-542, 2010.

[26] J. Shi and L.-L. Cui, "Characteristics and influencing factors of haze in the Yangtze River Delta region," China Environmental Science, vol. 33, no. 12, pp. 2113-2122, 2013.

[27] J. Song, T. Chen, Z. Q. Xie, and Q. Miao, "Impact on spatiotemporal variation of fog and haze days due to rapid urbanization in Jiangsu," Journal of the Meteorological Sciences, vol. 32, no. 3, pp. 275-281, 2012.

[28] X.-L. Chen, Y.-R. Feng, A.-Y. Wang et al., "Numerical experiment research on air pollutants during atmospheric haze over the multi-cities of pearl river delta region," Acta Scientiarum Natralium Universitatis Sunyatseni (Chinese Edition), vol. 46, no. 4, pp. 103-107, 2007.
[29] D. Wu, G. L. Liao, X. J. Deng et al., "Transport condition of surface layer under haze weather over the Pearl River Delta," Journal of Applied Meteorological Science, vol. 19, no. 1, pp. 1-9, 2008 (Chinese).

[30] H. H. Chen, D. Wu, H. B. Tan et al., "Study on the character of haze weather process from the year 2001 to 2008 over the Pearl River Delta," Journal of Tropical Meteorology (Chinese Edition), vol. 26, no. 2, pp. 147-155, 2010.

[31] P. S. Zhao, X. F. Xu, W. Meng et al., "Characteristics of hazy days in the region of Beijing, Tianjin, and Hebei," China Environmental Science, vol. 32, no. 1, pp. 31-36, 2012.

[32] X. Q. Wang, M. S. Sun, T. Yang et al., "Interdecadal change in frequency of dust-haze episodes in north China plain," Climatic and Environmental Research (Chinese Edition), vol. 18, no. 2, pp. 165-170, 2013.

[33] Z. H. Ren, F. Q. Su, Z. H. Chen et al., "Influence of synoptic systems on the distribution and evolution process of PM10 concentration in the boundary layer in summer and autumn," Chinese Journal of Atmospheric Sciences, vol. 32, no. 4, pp. 741751, 2008 (Chinese).

[34] X. Q. Rao, F. Li, N. F. Zhou et al., "Analysis of a large-scale haze over middle and eastern China," Meteorological Monthly (Chinese Edition), vol. 34, no. 6, pp. 89-96, 2008.

[35] P. B. Zhang, A. J. Jiang, J. L. Sun, Y. Jiang, P. He, and D. Liu, "Decadal change characteristics of autumn haze days in Jiangsu Province and its influential factors," Advances in Climate Change Research, vol. 8, no. 3, pp. 205-212, 2012 (Chinese).

[36] J.-C. Duan, X.-H. Bi, J.-H. Tan, G.-Y. Sheng, and J.-M. Fu, "Particle diameter distribution of polycyclic aromatic hydrocarbons (PAHs) in atmospheric particle during haze period in Guangzhou," China Environmental Science (Chinese Edition), vol. 26, no. 1, pp. 6-10, 2006.

[37] Y.-Q. Tong, Y. Yin, L. Qian, and J.-L. An, "Analysis of the characteristics of hazy phenomena in Nanjing area," China Environmental Science, vol. 27, no. 5, pp. 584-588, 2007 (Chinese).

[38] W. B. Tang, "The climatic characteristics of haze and the influence of heat island effect in Guilin in the past 50 years," Journal of Meteorological Research and Application (Chinese Edition), vol. 28, no. 4, pp. 20-25, 2007.

[39] J. P. Zhao, J. H. Tan, X. H. Bi et al., "The mass concentrations of inorganic elements in atmospheric particles during haze period in Guangzhou," Environmental Chemistry (Chinese Edition), vol. 27, no. 3, pp. 322-326, 2008.

[40] Y. Y. Guo, A. M. Miao, and H. Y. Zhang, "Analysis on the climate characteristics of the haze-weather for nearly 47 years in Taiyuan City," Sci-Tech Information Development \& Economy (Chinese Edition), vol. 18, no. 33, pp. 120-121, 2008.

[41] J. Guo, "Climatic characteristics of atmospheric haze in Tianjin area," Urban Environment \& Urban Ecology (Chinese Edition), vol. 2, no. 3, pp. 12-14, 2008.

[42] N. F. Zhou, F. Li, X. Q. Rao, and K. Yang, "Study on haze weather in China during winter-time of 2006," Meteorological Monthly, vol. 34, no. 6, pp. 81-88, 2008 (Chinese).

[43] H. Pan, F. Geng, Y. Chen et al., "Analysis of a haze event by micro-pulse light laser detection and ranging measurements in Shanghai," Acta Scientiae Circumstantiae, vol. 30, no. 11, pp. 2164-2173, 2010.

[44] H. G. Xu, S. Deng B, X. G. Zhou et al., "Effect of fog on urban boundary layer and environment," Journal of Applied Meteorological Science (Chinese Edition), vol. 13, no. 1, pp. 170176, 2002. 
[45] Z. L. Wu, A. X. Liu, C. C. Zhang et al., "Vertical distribution feature of PM2.5 and effect of boundary layer in Tianjin," Urban Environment \& Urban Ecology (Chinese Edition), vol. 22, no. 4, pp. 24-29, 2009.

[46] T. Y. Xiu, Y. Sun, T. Song, and Y. Wang, "The vertical distribution of ozone and boundary layer structure analysis during summer haze in Beijing," Acta Scientiae Circumstantiae (Chinese Edition), vol. 33, no. 2, pp. 321-331, 2013.

[47] M. Xu, Y.-Q. Zhu, and W.-T. Yu, "Preliminary study on haze in Tianjin," The Chinese Journal of Process Engineering, vol. 6, no. 2, pp. 127-132, 2006.

[48] X. Zhang, X. H. Cai, and F. H. Chai, "Structures and characteristics of the atmospheric boundary layer over Beijing area in autumn," Acta Scientiarum Naturalium Universitatis Pekinensis, vol. 42, no. 2, pp. 220-225, 2006.

[49] J. W. Yu, Y. Sun, B. Zhang et al., "Analysis of one heavy haze case happened in the region around Yangtze River in Jiangsu province," Journal of the Meteorological Sciences (Chinese Edition), vol. 29, no. 5, pp. 664-669, 2009.

[50] H. Zhang, C. E. Shi, M. Y. Qiu, and W. Xie, "Long-term variation of haze phenomena in Heifei and its impact factors," Acta Scientiae Circumstantiae (Chinese Edition), vol. 30, no. 4, pp. 714-721, 2010.

[51] Y. H. Zhang, Y. J. Ma, N. W. Liu, D. Zhou, Y. Wang, and Y. Hong, "Study on environmental characteristics of haze in Shenyang," Ecology and Environmental Sciences, vol. 19, no. 11, pp. 26362641, 2010 (Chinese).

[52] X. L. Zhang and R. H. Chen, "Inversion characteristic analysis of Nanyang boundary layer," Meteorological and Environmental Sciences (Chinese Edition), vol. 34, no. S1, pp. 116-119, 2011.

[53] J.-L. Zhu, T.-J. Wang, L. Xing, Q. Mu, and D.-R. Zhou, “Analysis on the characteristics and mechanism of a heavy haze episode in Jiangsu Province," China Environmental Science (Chinese Edition), vol. 31, no. 12, pp. 1943-1950, 2011.

[54] Y. Zhang, Y. Yin, L. X. Shi et al., "An observational study of aerosol property under typical polluted weather condition," Plateau Meteorology (Chinese Edition), vol. 31, no. 5, pp. 14321438, 2012.

[55] J. Zhang and S. T. Rao, "The role of vertical mixing in the temporal evolution of ground-level ozone concentrations," Journal of Applied Meteorology, vol. 38, no. 12, pp. 1674-1691, 1999.

[56] P. Solomon, E. Cowling, G. Hidy, and C. Furiness, "Comparison of scientific findings from major ozone field studies in North America and Europe," Atmospheric Environment, vol. 34, no. 1214, pp. 1885-1920, 2000.

[57] G. M. Hidy, "Ozone process insights from field experimentspart I: overview," Atmospheric Environment, vol. 34, no. 12-14, pp. 2001-2022, 2000.

[58] C.-H. Lin, Y.-L. Wu, C.-H. Lai, P.-H. Lin, H.-C. Lai, and P.L. Lin, "Experimental investigation of ozone accumulation overnight during a wintertime ozone episode in south Taiwan," Atmospheric Environment, vol. 38, no. 26, pp. 4267-4278, 2004.

[59] J. E. Yorks, A. M. Thompson, E. Joseph, and S. K. Miller, "The variability of free tropospheric ozone over Beltsville, Maryland (39N, 77W) in the summers 2004-2007," Atmospheric Environment, vol. 43, no. 11, pp. 1827-1838, 2009.

[60] Y. F. Guo, D. Y. Liu, B. Zhou et al., "Study on haze characteristics in Wuxi and its impact factors," Meteorological Monthly, vol. 39, no. 10, pp. 1314-1324, 2013.
[61] China Meteorological Administration, "Observation and prediction of haze," the meteorological industry standard of the People's Republic of China, QX/T113-2010, 2010.

[62] M. Tao, L. Chen, Z. Wang, J. Tao, and L. Su, "Satellite observation of abnormal yellow haze clouds over East China during summer agricultural burning season," Atmospheric Environment, vol. 79, pp. 632-640, 2013.

[63] G. Wang, K. Kakawamura, M. Xie et al., "Organic molecular compositions and size distributions of Chinese summer and autumn aerosols from Nanjing: characteristic haze event caused by wheat straw burning," Environmental Science \& Technology, vol. 43, no. 17, pp. 6493-6499, 2009.

[64] G.-L. Cao, X.-Y. Zhang, D. Wang, and F.-C. Zheng, "Inventory of atmospheric pollutants discharged from biomass burning in China continent," China Environmental Science, vol. 25, no. 4, pp. 389-393, 2005. 

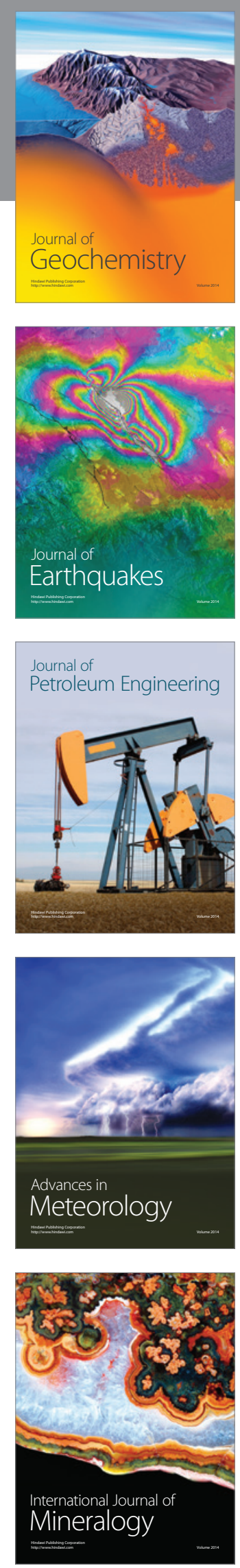
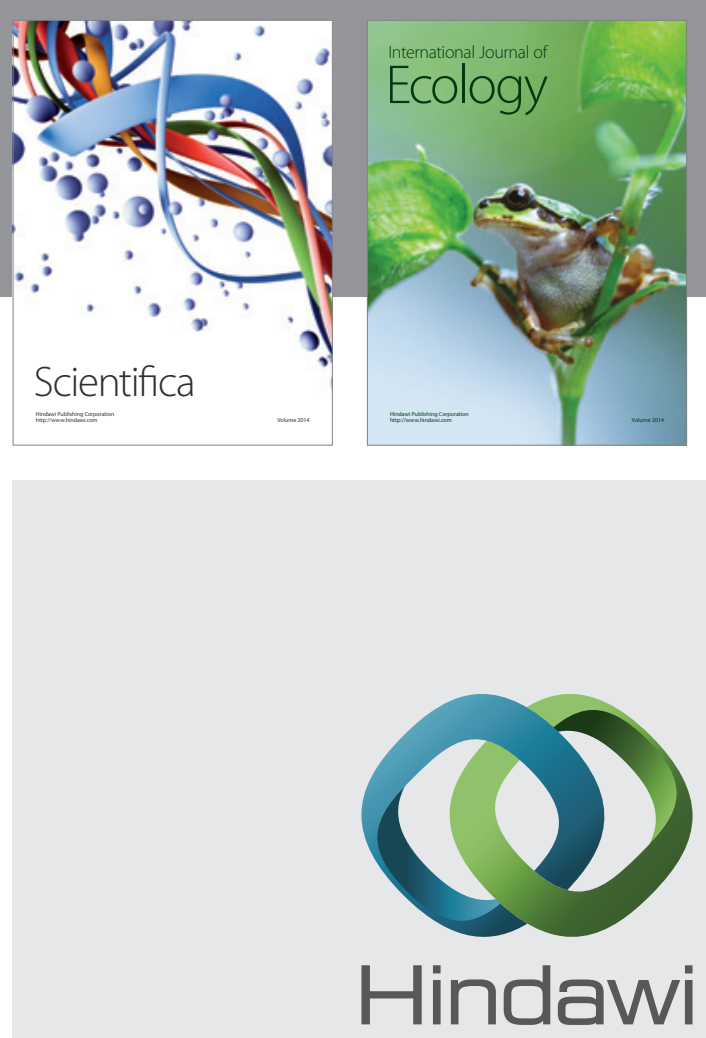

Submit your manuscripts at

http://www.hindawi.com
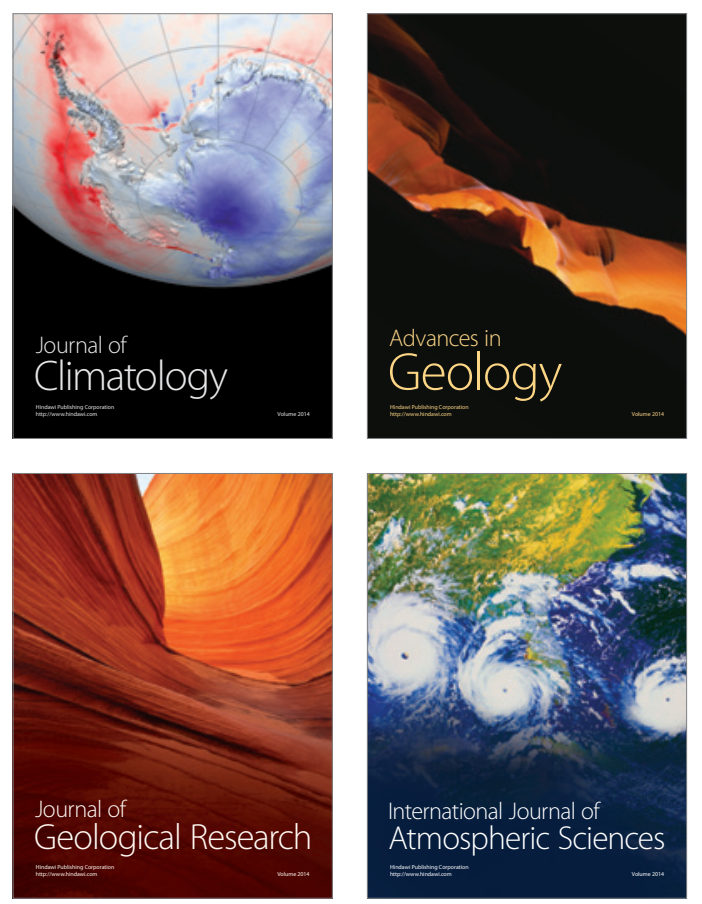

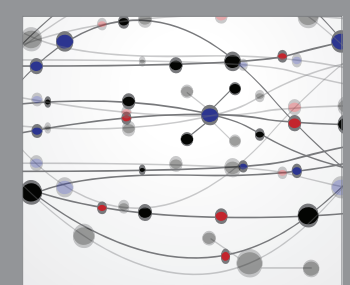

The Scientific

\section{World Journal}
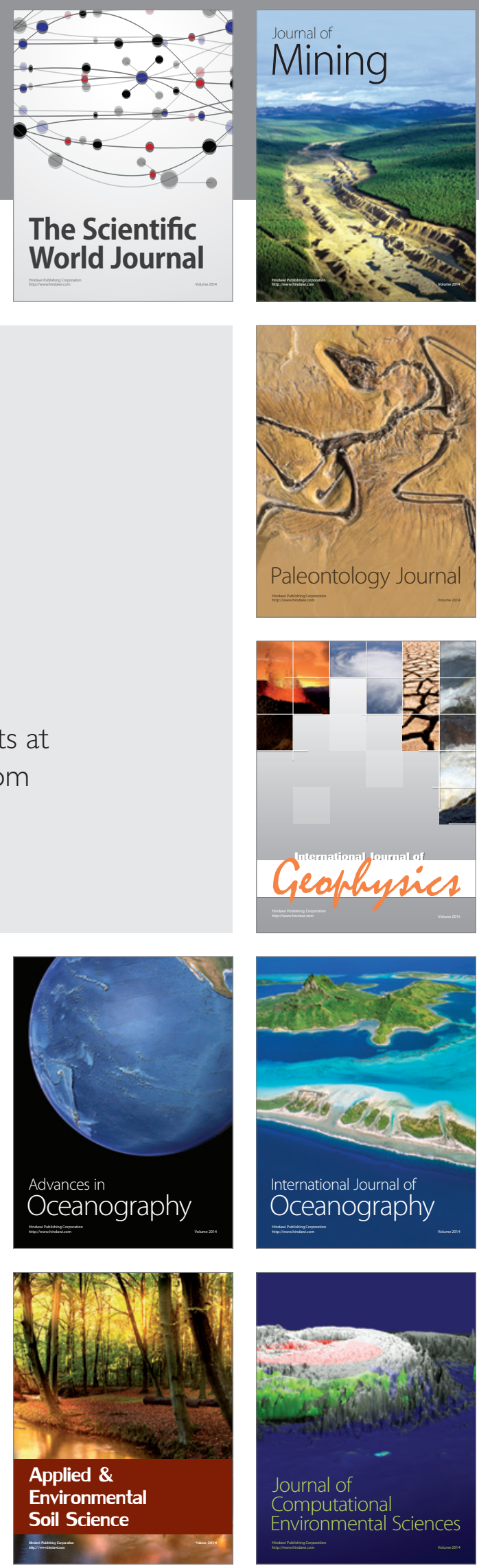